\title{
Comparison of Twelve Types of Rough Approximations Based on j-Neighborhood Space and j-Adhesion Neighborhood Space
}

Mohamed Atef ( $\nabla$ matef@science.menofia.edu.eg )

Menoufia University https://orcid.org/0000-0001-9439-3404

Ahmed Mostafa Khalil

Al-Azhar University Faculty of Science

Abdelfatah Azzam

New Vally University

Abd El Fattah El Atik

Tanta University

Sheng Gang Li

Shaanxi Normal University

Heng Liu

Guangxi University for Nationalities

\section{Research Article}

Keywords: Rough sets, Lower and upper approximations, j Neighborhood space, j-Adhesion neighborhood space, Accuracy measure

Posted Date: August 26th, 2021

DOl: https://doi.org/10.21203/rs.3.rs-664569/v1

License: (c) (1) This work is licensed under a Creative Commons Attribution 4.0 International License.

Read Full License

Version of Record: A version of this preprint was published at Soft Computing on November 2nd, 2021.

See the published version at https://doi.org/10.1007/s00500-021-06426-5. 


\title{
Comparison of twelve types of rough approximations based on $j$-neighborhood space and $j$-adhesion neighborhood space
}

\author{
Mohammed Atef $^{1} \cdot$ Ahmed Mostafa Khalil $^{2 *} \cdot$ Sheng-Gang Li $^{3} \cdot$ Abdelfatah Azzam $^{4} \cdot$ Heng \\ Liu $^{5}$ - Abd El Fattah El Atik ${ }^{6}$
}

Received: date / Accepted: date

\begin{abstract}
In this paper, we generalize six kinds of rough set models based on $j$-neighborhood space (i.e., reflexive $1 j$-neighborhood rough set, reflexive $2 j$-neighborhood rough set, reflexive $3 j$ neighborhood rough set, similarity $4 j$-neighborhood rough set, similarity $5 j$-neighborhood rough set, and similarity $6 j$-neighbor hood rough set), and investigate some of their basic properties. Further, we propose a new neighborhood space called j-adhesion neighborhood based on six types of rough set models (i.e., reflexive $7 j$-adhesion neighborhood rough set, reflexive $8 j$-adhesion neighborhood rough set, reflexive $9 j$-adhesion neighborhood rough set, similarity $10 j$-adhesion neighborhood rough set, similarity $11 j$-adhesion neighborhood rough set, and similarity $12 j$-neighbor hood rough set) to reduce the boundary region and the accuracy. The fundamental properties of approximation operators based on $j$-adhesion neighborhood space are investigated. The relationship between the properties of these types is explained. Finally, we give comparisons between the proposed approach with the previous approach (i.e., Abo-Tabl's approach and Dai et al.'s approach)
\end{abstract}

\footnotetext{
Mohammed Atef

Email: matef@science.menofia.edu.eg

Ahmed Mostafa Khalil

Email: a.khalil@azhar.edu.eg

Sheng-Gang Li

Email:shengganglinew@126.com

Abdelfatah Azzam

Email: azzam0911@yahoo.com

Heng Liu

Email: liuheng122@gmail.com

Abd El Fattah El Atik

Email: aelatik55@yahoo.com

${ }^{1}$ Department of Mathematics and Computer Science, Faculty of Science, Menoufia University, Menoufia 32511, Egypt

${ }^{2}$ Department of Mathematics, Faculty of Science, Al-Azhar University, Assiut 71524, Egypt

${ }^{3}$ College of Mathematics and Information Science, Shaanxi Normal University, Xi'an 710062, China

${ }^{4}$ Department of Mathematics, Faculty of Science, New Valley University, Elkharga 72511, Egypt

${ }^{5}$ School of Science, Guangxi University for Nationalities, Nanning 530006, China

${ }^{6}$ Department of Mathematics, Faculty of Science, Tanta University, Tanta 31511, Egypt .
}

from six types of rough set models. Consequently, the accuracy from the proposed approach is improved.

Keywords Rough sets. Lower and upper approximations. $j$ Neighborhood space. $j$-Adhesion neighborhood space. Accuracy measure

\section{Introduction}

The original concept of a rough set is proposed by Pawlak [1, 2]. The rough set theory has been applied to many different fields, such as economics, engineering, medicine, biology, chemistry, banking, market research, speech recognition, information analysis, data analysis, material science, data mining, networking, linguistics, and other fields (see, e.g., [3-15]). A framework for the formulation, interpretation, and comparison of neighborhood systems and rough set approximations (lower / upper) using the more familiar notion of binary relations is given by Yao [16]. He also introduced a special class of neighborhood systems, it is called 1neighborhood systems and analyzed three extensions of Pawlak's lower and upper approximations. Later, many researchers presented some concepts based on a rough set, for example, similarity relation [17], the tolerance relation [18], and the arbitrary binary relation [16, 19-22]. Abu-Donia [23] discussed three types of lower and upper approximations of any set concerning any relationship based on the right neighborhood and generalized these three types of approximations into two ways by using a finite number of any binary relations. Abo-Tabl [24] gave a comparison of two types of definitions of the rough approximations based on a similarity relation (i.e., reflexivity and symmetry). Besides, he defined three new types of generalized rough approximations. Dai et al. [25] determined the generalized rough set models by multiple neighborhoods generated from a similarity relation.

In 2014, Abd El-Monsef et al. [26] presented the main ideas about the concept of " $j$-neighborhood space"and studied eight approaches for approximating rough sets. Abbas et al. [27] introduced the new concepts of $j$-near closure operators induced from binary relation and its applications. Hosny [28] gave a generalization of rough sets by using two different methods. Abd El-Monsef [29] presented the cover rough sets based on $j$-neighborhoods by approximation operations as a new type of extended covering 
rough set models. Amer [30] proposed the concepts of " $j$-near" in rough sets with some applications.

Regarding these developments, as the motivation of this paper, we will generalize the three types of Abo-Tabl's lower and upper approximations and three types of Dai et al.'s lower and upper approximations of any set concerning for $j$-neighborhood space. Further, we will define six new types of approximations based on $j$-adhesion neighborhood space. Properties of approximation operators based on $j$-adhesion neighborhood space are explained and discussed. The relationship between these types of the $j$-adhesion neighborhood space is introduced. Finally, we will give a comparison between the previous approach (i.e., AboTabl's approach [24] and Dai et al.'s approach [25]) and our approach by using $j$-adhesion neighborhood space.

The rest of this paper is organized as follows. In Section 2, we review some basic concepts, such as types of rough set approximations based on binary relation, $j$-neighborhood of element $x$, $j$-neighborhood space, Abo-Tabl's approach, and Dai et al.'s approach. In Section 3, we generalize six types of rough sets based on $j$-neighborhood space. Several of the existing properties of six types of rough sets are explained. A new six types of rough set based on $j$-adhesion neighborhood space are defined and investigated their properties are shown in Section 4. A comparison between the previous approach (i.e., Abo-Tabl's approach [24] and Dai et al.'s approach [25]) and our approach is discussed in Section 5. Finally, conclusions are given in Section 6.

\section{Preliminaries}

In this section, we recall several basic notions and results which will be used in this paper as indicated below.

\subsection{Types of rough set approximations based on binary re-} lation

The concept of a rough set to any relation $\Re$ extended by Yao [16] as follows:

Definition 2.1 (cf. [16]). Let $\Re$ be an arbitrary binary relation on an universe set $\mathcal{U}$ and $(\mathcal{U}, \Re)$ be an approximation space. Then

$$
\Re(u)=\{v \in \mathcal{U}: u \Re v\} .
$$

Definition 2.2 (cf. [16]). Let $(\mathcal{U}, \Re)$ be an approximation space and $\Re \sqsubseteq \mathcal{U} \times \mathcal{U}$. The lower and the upper approximations of $\hat{X} \sqsubseteq \mathcal{U}$ in $(\mathcal{U}, \Re)$ or with respect to $\Re$ is a pair $\left(\Re^{-}(\hat{X}), \Re^{+}(\hat{X})\right)$, defined by

$$
\Re^{-}(\hat{X})=\{u \in \mathcal{U}: \Re(u) \sqsubseteq \hat{X}\}
$$

(called the lower approximation of $\hat{X}$ ) and

$$
\Re^{+}(\hat{X})=\{u \in \mathcal{U}: \Re(u) \sqcap \hat{X} \neq \emptyset\}
$$

(called the upper approximation of $\hat{X}$ ).

We list the properties that are of interest in the theory of rough sets, let $\hat{X}, \hat{X}_{1}, \hat{X}_{2} \sqsubseteq \mathcal{U}$ :

(L1) $\Re^{-}(\hat{X})=\left(\Re^{+}\left(\hat{X}^{c}\right)\right)^{c}$.

(L2) $\Re^{-}(\mathcal{U})=\mathcal{U}$.

(L3) $\Re^{-}\left(\hat{X}_{1} \sqcap \hat{X}_{2}\right)=\Re^{-}\left(\hat{X}_{1}\right) \sqcap \Re^{-}\left(\hat{X}_{2}\right)$.

(L4) $\Re^{-}\left(\hat{X}_{1}\right) \sqcup \Re^{-}\left(\hat{X}_{2}\right) \sqsubseteq \Re^{-}\left(\hat{X}_{1} \sqcup \hat{X}_{2}\right)$.

(L5) If $\hat{X}_{1} \sqsubseteq \hat{X}_{2}$, then $\Re^{-}\left(\hat{X}_{1}\right) \sqsubseteq \Re^{-}\left(\hat{X}_{2}\right)$.
(L6) $\Re^{-}(\emptyset)=\emptyset$.

(L7) $\Re^{-}(\hat{X}) \sqsubseteq \hat{X}$.

(L8) $\hat{X} \sqsubseteq \Re^{-}\left(\Re^{+}(\hat{X})\right)$.

(L9) $\Re^{-}\left(\Re^{-}(\hat{X})\right)=\Re^{-}(\hat{X})$.

(L10) $\Re^{+}(\hat{X}) \sqsubseteq \Re^{-}\left(\Re^{+}(\hat{X})\right)$.

(U1) $\Re^{+}(\hat{X})=\left(\Re^{-}\left(\hat{X}^{c}\right)\right)^{c}$.

(U2) $\Re^{+}(\emptyset)=\emptyset$.

(U3) $\Re^{+}\left(\hat{X}_{1} \sqcap \hat{X}_{2}\right) \sqsubseteq \Re^{+}\left(\hat{X}_{1}\right) \sqcap \Re^{+}\left(\hat{X}_{2}\right)$.

(U4) $\Re^{+}\left(\hat{X}_{1} \sqcup \hat{X}_{2}\right)=\Re^{+}\left(\hat{X}_{1}\right) \sqcup \Re^{+}\left(\hat{X}_{2}\right)$.

(U5) If $\hat{X}_{1} \sqsubseteq \hat{X}_{2}$, then $\Re^{+}\left(\hat{X}_{1}\right) \sqsubseteq \Re^{+}\left(\hat{X}_{2}\right)$.

(U6) $\Re^{+}(\mathcal{U})=\mathcal{U}$.

(U7) $\hat{X} \sqsubseteq \Re^{+}(\hat{X})$.

(U8) $\Re^{+}\left(\Re^{-}(\hat{X})\right) \sqsubseteq \hat{X}$.

(U9) $\Re^{+}\left(\Re^{+}(\hat{X})\right)=\Re^{+}(\hat{X})$.

(U10) $\Re^{+}\left(\Re^{-}(\hat{X})\right) \sqsubseteq \Re^{-}(\hat{X})$.

(K) $\Re^{-}\left(\hat{X}_{1}^{c} \sqcup \hat{X}_{2}\right) \sqsubseteq\left(\Re^{-}\left(\hat{X}_{1}\right)\right)^{c} \sqcup \Re^{-}\left(\hat{X}_{2}\right)$.

(LU) $\Re^{-}(\hat{X}) \sqsubseteq \Re^{+}(\hat{X})$.

\subsection{Types of rough set approximations based on Abo-Tabl's} approach [24]

Abo-Tabl [24] presented the following definitions:

Definition 2.3. Let $\Re$ be any reflexive relation on a universe set $\mathcal{U}$ and $(\mathcal{U}, \Re)$ be an approximation space. The set $\Re \sqcap(u)(\forall u \in$ $\mathcal{U})$ (The intersection of all right neighborhoods containing $u$ ) as follows:

$$
\Re \sqcap(u)=\left\{\begin{array}{ll}
\bigcap_{u \in \Re(v)} \Re(v), \text { if } \exists v, u \in \Re(v) \\
\emptyset, \quad \text { otherwise }
\end{array} .\right.
$$

Definition 2.4. Let $(\mathcal{U}, \Re)$ be a reflexive approximation space and $\Re \sqsubseteq \mathcal{U} \times \mathcal{U}$. The first type of the reflexive lower and reflexive upper approximations of $\hat{X} \sqsubseteq \mathcal{U}$ in $(\mathcal{U}, \Re)$ or with respect to $\Re$ is a pair $\left(\Re^{-}(\hat{X}), \Re^{+}(\hat{X})\right)$, defined by

$$
\Re^{-}(\hat{X})=\left\{u \in \mathcal{U}: \Re^{\sqcap}(u) \sqsubseteq \hat{X}\right\}
$$

(called the first reflexive lower approximation of $\hat{X}$ ) and

$$
\Re^{+}(\hat{X})=\{u \in \mathcal{U}: \Re \sqcap(u) \sqcap \hat{X} \neq \emptyset\}
$$

(called the first reflexive upper approximation of $\hat{X}$ ).

Definition 2.5. Let $(\mathcal{U}, \Re)$ be a reflexive approximation space and $\Re \sqsubseteq \mathcal{U} \times \mathcal{U}$. The second type of the reflexive lower and reflexive upper approximations of $\hat{X} \sqsubseteq \mathcal{U}$ in $(\mathcal{U}, \Re)$ or with respect to $\Re$ is a pair $\left(\Re^{-}(\hat{X}), \Re^{+}(\hat{X})\right)$, defined by

$$
\Re^{-}(\hat{X})=\bigsqcup\left\{\Re \sqcap(u): \Re^{\sqcap}(u) \sqsubseteq \hat{X}\right\}
$$

(called the second reflexive lower approximation of $\hat{X}$ ) and

$$
\Re^{+}(\hat{X})=\left[\Re^{-}\left(\hat{X}^{c}\right)\right]^{c}
$$

(called the second reflexive upper approximation of $\hat{X}$ ).

Definition 2.6. Let $(\mathcal{U}, \Re)$ be a reflexive approximation space and $\Re \sqsubseteq \mathcal{U} \times \mathcal{U}$. The third type of the reflexive lower and reflexive upper approximations of $\hat{X} \sqsubseteq \mathcal{U}$ in $(\mathcal{U}, \Re)$ or with respect to $\Re$ is a pair $\left(\Re^{-}(\hat{X}), \Re^{+}(\hat{X})\right)$, defined by

$$
\Re^{-}(\hat{X})=\left[\Re^{+}\left(\hat{X}^{c}\right)\right]^{c}
$$


(called the third reflexive lower approximation of $\hat{X}$ ) and

$$
\Re^{+}(\hat{X})=\bigsqcup\{\Re \sqcap(u): \Re \sqcap(u) \sqcap \hat{X} \neq \emptyset\}
$$

(called the third reflexive upper approximation of $\hat{X}$ ).

\subsection{Types of rough set approximations based on Dai et al.'s approach [25]}

Dai et al. [25] introduced the following definitions:

Definition 2.7. Let $\Re$ be a similarity relation on a universe set $\mathcal{U}$ and $(\mathcal{U}, \Re)$ be an approximation space. The set $\Re^{\sqcup}(u)(\forall u \in$ $\mathcal{U}$ ) (The union of all right neighborhoods containing $u$ ) as follows:

$$
\Re^{\sqcup}(u)=\left\{\begin{array}{ll}
\bigsqcup_{u \in \Re(v)} \Re(v), \text { if } \exists v, u \in \Re(v) \\
\emptyset, & \text { otherwise }
\end{array} .\right.
$$

Definition 2.8. Let $(\mathcal{U}, \Re)$ be a similarity approximation space and $\Re \sqsubseteq \mathcal{U} \times \mathcal{U}$. The first type of the similarity lower and similarity upper approximations of $\hat{X} \sqsubseteq \mathcal{U}$ in $(\mathcal{U}, \Re)$ or with respect to $\Re$ is a pair $\left(\Re^{-}(\hat{X}), \Re^{+}(\hat{X})\right)$, defined by

$$
\Re^{-}(\hat{X})=\left\{u \in \mathcal{U}: \Re^{\sqcup}(u) \sqsubseteq \hat{X}\right\}
$$

(called the first similarity lower approximation of $\hat{X}$ ) and

$$
\Re^{+}(\hat{X})=\left\{u \in \mathcal{U}: \Re^{\sqcup}(u) \sqcap \hat{X} \neq \emptyset\right\}
$$

(called the first similarity upper approximation of $\hat{X}$ ).

Definition 2.9. Let $(\mathcal{U}, \Re)$ be a similarity approximation space and $\Re \sqsubseteq \mathcal{U} \times \mathcal{U}$. The second type of the similarity lower and similarity upper approximations of $\hat{X} \sqsubseteq \mathcal{U}$ in $(\mathcal{U}, \Re)$ or with respect to $\Re$ is a pair $\left(\Re^{-}(\hat{X}), \Re^{+}(\hat{X})\right)$, defined by

$$
\Re^{-}(\hat{X})=\bigsqcup\left\{\Re^{\sqcup}(u): \Re^{\sqcup}(u) \sqsubseteq \hat{X}\right\}
$$

(called the second similarity lower approximation of $\hat{X}$ ) and

$$
\Re^{+}(\hat{X})=\left[\Re^{-}\left(\hat{X}^{c}\right)\right]^{c}
$$

(called the second similarity upper approximation of $\hat{X}$ ).

Definition 2.10. Let $(\mathcal{U}, \Re)$ be a similarity approximation space and $\Re \sqsubseteq \mathcal{U} \times \mathcal{U}$. The third type of the similarity lower and similarity upper approximations of $\hat{X} \sqsubseteq \mathcal{U}$ in $(\mathcal{U}, \Re)$ or with respect to $\Re$ is a pair $\left(\Re^{-}(\hat{X}), \Re^{+}(\hat{X})\right)$, defined by

$$
\Re^{-}(\hat{X})=\left[\Re^{+}\left(\hat{X}^{c}\right)\right]^{c}
$$

(called the third similarity lower approximation of $\hat{X}$ ) and

$$
\Re^{+}(\hat{X})=\bigsqcup\left\{\Re^{\sqcup}(u): \Re^{\sqcup}(u) \sqcap \hat{X} \neq \emptyset\right\}
$$

(called the third similarity upper approximation of $\hat{X}$ ).

\section{4.j-Neighborhood of element $u$ and j-neighborhood space}

Abd El-Monsef et al. [26] proposed the concepts of $j$-neighborhood of an element $u$ and $j$-neighborhood space as indicated below.
Definition 2.11. Let $\Re$ be an arbitrary binary relation on $\mathcal{U}$. The $j$-neighborhood of an element $u \in \mathcal{U}$ are defined as follows: $\forall j \in\left\{j_{1}, j_{2}, j_{3}, j_{4}, j_{5}, j_{6}, j_{7}, j_{8}\right\}$

(1) $\aleph_{j_{1}}(u)=\{v \in \mathcal{U} \mid u \Re v\}$.

(2) $\aleph_{j_{2}}(u)=\{v \in \mathcal{U} \mid v \Re u\}$.

(3) $\aleph_{j_{3}}(u)=\left\{v \in \mathcal{U} \mid \bigcap_{u \in \aleph_{j_{1}}(v)} \aleph_{j_{1}}(v)\right\}$.

(4) $\aleph_{j_{4}}(u)=\left\{v \in \mathcal{U} \mid \bigcap_{u \in \aleph_{j_{2}}(v)} \aleph_{j_{2}}(v)\right\}$.

(5) $\aleph_{j_{5}}(u)=\aleph_{j_{1}}(u) \sqcup \aleph_{j_{2}}(u)$.

(6) $\aleph_{j_{6}}(u)=\aleph_{j_{1}}(u) \sqcap \aleph_{j_{2}}(u)$.

(7) $\aleph_{j_{7}}(u)=\aleph_{j_{3}}(u) \sqcup \aleph_{j_{4}}(u)$.

(8) $\aleph_{j_{8}}(u)=\aleph_{j_{3}}(u) \sqcap \aleph_{j_{4}}(u)$.

Definition 2.12. Let $\Re$ be an arbitrary binary relation on $\mathcal{U}$, $2^{\mathcal{U}}$ the set of all subset of $\mathcal{U}$, and $\vartheta_{j}: \mathcal{U} \rightarrow 2^{\mathcal{U}}$ be a mapping which assigns for each $u$ in $\mathcal{U}$ its $j$-neighborhood in $2^{\mathcal{U}}$. The triple $\left(\mathcal{U}, \Re, \vartheta_{j}\right)$ is called a $j$-neighborhood space (briefly, $j$-NSS).

Remark 2.13. (1) If $\Re$ be any reflexive relation on a universe set $\mathcal{U}$, then the triple $\left(\mathcal{U}, \Re, \vartheta_{j}\right)$ is called a reflexive $j$-neighborhood space (briefly, $j$-RNS).

(2) If $\Re$ be any similarity relation on a universe set $\mathcal{U}$, then the triple $\left(\mathcal{U}, \Re, \vartheta_{j}\right)$ is called a similarity $j$-neighborhood space (briefly, $j$-SNS).

3 Generalized rough set models by Abo-Tabl's approach [24] and Dai et al.'s approach [25] based on j-neighborhood space

\subsection{First kind: Generalized rough set models by Abo-Tabl's ap- proach [24] based on j-reflexive neighborhood space}

In this subsection, we will extend the Definitions 2.3, 2.4, 2.5 and 2.6 to $j$-reflexive neighborhood space as indicated below.

Definition 3.1. Let $\Re$ be any reflexive relation on a universe set $\mathcal{U}$ and $\left(\mathcal{U}, \Re, \vartheta_{j}\right)$ be a $j$ - $\mathbb{R N S}$. We define a set $\aleph_{j}^{\Gamma}(u)(\forall u \in$ $\mathcal{U}$ ) (The intersection of all $j$-neighborhoods containing $u$ ) as follows:

$$
\aleph_{j}^{\sqcap}(u)= \begin{cases}\bigcap_{u \in \aleph_{j}(v)} \aleph_{j}(v), \text { if } \exists v, u \in \aleph_{j}(v) \\ \emptyset, \quad \text { otherwise }\end{cases}
$$

Example 3.2. Let $\mathcal{U}=\{1,2,3,4\}$ and $\Re \sqsubseteq \mathcal{U} \times \mathcal{U}$ be a reflexive relation on $\mathcal{U}$ defined by

$$
\Re=\{(1,1),(2,2),(3,3),(4,4),(1,2),(1,3),(2,3),(3,4),
$$

Firstly, we calculate the $j$-neighborhood according to Definition 2.11 as follows $\left(\forall u \in U, \forall j \in\left\{j_{1}, j_{2}, j_{3}, j_{4}, j_{5}, j_{6}, j_{7}, j_{8}\right\}\right)$ :

(1) Take $j \in\left\{j_{1}, j_{4}\right\}$, we get

$$
\begin{gathered}
\aleph_{j}(1)=\{1,2,3\}, \aleph_{j}(2)=\{2,3\}, \\
\aleph_{j}(3)=\{3,4\}, \aleph_{j}(4)=\{2,4\} .
\end{gathered}
$$

(2) Take $j \in\left\{j_{2}, j_{3}\right\}$, we get

$$
\begin{gathered}
\aleph_{j}(1)=\{1\}, \aleph_{j}(2)=\{1,2,4\}, \\
\aleph_{j}(3)=\{1,2,3\}, \aleph_{j}(4)=\{3,4\} .
\end{gathered}
$$


(3) Take $j \in\left\{j_{5}, j_{7}\right\}$, we get

$$
\begin{aligned}
& \aleph_{j}(1)=\{1,2,3\}, \aleph_{j}(2)=\mathcal{U}, \\
& \aleph_{j}(3)=\mathcal{U}, \aleph_{j}(4)=\{2,3,4\} .
\end{aligned}
$$

(4) Take $j \in\left\{j_{6}, j_{8}\right\}$, we get

$$
\begin{aligned}
& \aleph_{j}(1)=\{1\}, \aleph_{j}(2)=\{2\}, \\
& \aleph_{j}(3)=\{3\}, \aleph_{j}(4)=\{4\} .
\end{aligned}
$$

Secondly, we calculate the $\aleph_{j}^{\sqcap}(u)$ according to Definition 3.1 as follows $\left(\forall u \in U, \forall j \in\left\{j_{1}, j_{2}, j_{3}, j_{4}, j_{5}, j_{6}, j_{7}, j_{8}\right\}\right)$ :

(1) Take $j \in\left\{j_{1}, j_{4}\right\}$, we get

$$
\begin{gathered}
\aleph_{j}^{\Gamma}(1)=\{1,2,3\}, \quad \aleph_{j}^{\Gamma}(2)=\{2\}, \\
\aleph_{j}^{\Gamma}(3)=\{3\}, \aleph_{j}^{\Gamma_{j}}(4)=\{4\} .
\end{gathered}
$$

(2) Take $j \in\left\{j_{2}, j_{3}\right\}$, we get

$$
\begin{gathered}
\aleph_{j}^{\Pi}(1)=\{1\}, \aleph_{j}^{\Gamma}(2)=\{1,2\}, \\
\aleph_{j}^{\Pi}(3)=\{3\}, \aleph_{j}^{\Gamma}(4)=\{4\} .
\end{gathered}
$$

(3) Take $j \in\left\{j_{5}, j_{7}\right\}$, we get

$$
\begin{aligned}
& \aleph_{j}^{\Gamma}(1)=\{1,2,3\}, \aleph_{j}^{\Gamma}(2)=\{2,3\}, \\
& \aleph_{j}^{\Gamma}(3)=\{2,3\}, \aleph_{j}^{\Gamma_{j}}(4)=\{2,3,4\} .
\end{aligned}
$$

(4) Take $j \in\left\{j_{6}, j_{8}\right\}$, we get

$$
\begin{aligned}
& \aleph_{j}^{\Gamma}(1)=\{1\}, \quad \aleph_{j}^{\Gamma}(2)=\{2\}, \\
& \aleph_{j}^{\Gamma}(3)=\{3\}, \quad \aleph_{j}^{\Gamma}(4)=\{4\} .
\end{aligned}
$$

Definition 3.3. Let $\left(\mathcal{U}, \Re, \vartheta_{j}\right)$ be a $j$ - $\mathbb{R} \mathbb{S}$. A reflexive $1 j$ neighborhood rough set of $\hat{X} \sqsubseteq \mathcal{U}$ in $\left(\mathcal{U}, \Re, \vartheta_{j}\right)$ or with respect to $\vartheta_{j}$ is a pair $\left(\Re_{\aleph_{j}}^{-1}(\hat{X}), \Re_{\aleph_{j}}^{+1}(\overline{\hat{X}})\right)$, defined by

$$
\Re_{\aleph_{j}}^{-1}(\hat{X})=\left\{u \in \mathcal{U}: \aleph_{j}^{\Pi}(u) \sqsubseteq \hat{X}\right\}
$$

(called the reflexive 1-lower approximation of $\hat{X}$ ) and

$$
\Re_{\aleph_{j}}^{+1}(\hat{X})=\left\{u \in \mathcal{U}: \aleph_{j}^{\sqcap}(u) \sqcap \hat{X} \neq \emptyset\right\}
$$

(called the reflexive 1-upper approximation of $\hat{X}$ ).

Example 3.4 (Continued from Example 3.2). Let $\hat{X}=\{1,3\}$. Then

(1) Take $j \in\left\{j_{1}, j_{4}\right\}$, we get

$$
\Re_{\aleph_{j}}^{-1}(\hat{X})=\{3\}, \quad \Re_{\aleph_{j}}^{+1}(\hat{X})=\{1,3\} .
$$

(2) Take $j \in\left\{j_{2}, j_{3}\right\}$, we get

$$
\Re_{\aleph_{j}}^{-1}(\hat{X})=\{1,3\}, \quad \Re_{\aleph_{j}}^{+1}(\hat{X})=\{1,2,3\} .
$$

(3) Take $j \in\left\{j_{5}, j_{7}\right\}$, we get

$$
\Re_{\aleph_{j}}^{-1}(\hat{X})=\emptyset, \Re_{\aleph_{j}}^{+1}(\hat{X})=\mathcal{U}
$$

(4) Take $j \in\left\{j_{6}, j_{8}\right\}$, we get

$$
\Re_{\aleph_{j}}^{-1}(\hat{X})=\{1,3\}, \Re_{\aleph_{j}}^{+1}(\hat{X})=\{1,3\}
$$

Remark 3.5. When $j=j_{1}$ in Definition 3.3. Then we obtain on Definition 2.4.
Proposition 3.6. Let $\hat{X}, \hat{X}_{1}, \hat{X}_{2} \sqsubseteq \mathcal{U}$. Then the following properties (L1)-(L7), (L9), (U1)-(U7), (U9), (K), and (LU) will be satisfy based on Definition 3.3.

Proof. The following properties (L2), (L4), (L6), (L7), (U1)(U7), (U9) and (LU) follows from Definition 3.3.

(L1) As $\left[\Re_{\aleph_{j}}^{+1}\left(\hat{X}^{c}\right)\right]^{c}=\left\{u \in \mathcal{U}: \aleph_{j}^{\sqcap}(u) \sqcap \hat{X}^{c} \neq \emptyset\right\}^{c}=\{u \in$ $\left.\mathcal{U}: \aleph_{j}(u) \sqcap \hat{X}^{c}=\emptyset\right\}=\left\{u \in \mathcal{U}: \aleph_{j}(u) \sqsubseteq \hat{X}\right\}=\Re_{\aleph_{j}}^{-1}(\hat{X})$.

(L3) As $\Re_{\aleph_{j}}^{-1}\left(\hat{X}_{1} \sqcap \hat{X}_{2}\right)=\left\{u \in \mathcal{U}: \aleph_{j}^{\sqcap}(u) \sqsubseteq \hat{X}_{1} \sqcap \hat{X}_{2}\right\}=$ $\left\{u \in \mathcal{U}: \aleph_{j}^{\sqcap}(u) \sqsubseteq \hat{X}_{1} \wedge \aleph_{j}^{\Gamma}(u) \sqsubseteq \hat{X}_{2}\right\}=\left\{u \in \mathcal{U}: \aleph_{j}^{\sqcap}(u) \sqsubseteq\right.$ $\left.\hat{X}_{1}\right\} \sqcap\left\{u \in \mathcal{U}: \aleph_{j}^{\sqcap}(u) \sqsubseteq \hat{X}_{2}\right\}=\Re_{\aleph_{j}}^{-1}\left(\hat{X}_{1}\right) \sqcap \Re_{\aleph_{j}}^{-1}\left(\hat{X}_{2}\right)$.

(L5) Let $\hat{X}_{1} \sqsubseteq \hat{X}_{2}$ and $u \in \Re_{\aleph_{j}}^{-1}\left(\hat{X}_{1}\right)$. Then $\aleph_{j}^{\sqcap}(u) \sqsubseteq \hat{X}_{1}$ and so $\aleph_{j}^{\sqcap}(u) \sqsubseteq \hat{X}_{2}$ implies $u \in \Re_{\aleph_{j}}^{-1}\left(\hat{X}_{2}\right)$. Thus $\Re_{\aleph_{j}}^{-1}\left(\hat{X}_{1}\right) \sqsubseteq$ $\Re_{\aleph_{j}}^{-1}\left(\hat{X}_{2}\right)$.

(L9) By (L7), we obtain $\Re_{\aleph_{j}}^{-1}\left(\Re_{\aleph_{j}}^{-1}(\hat{X})\right) \sqsubseteq \Re_{\aleph_{j}}^{-1}(\hat{X})$. Now, we show $\Re_{\aleph_{j}}^{-1}(\hat{X}) \sqsubseteq \Re_{\aleph_{j}}^{-1}\left(\Re_{\aleph_{j}}^{-1}(\hat{X})\right)$. As $\aleph_{j}^{\Pi}(u) \sqsubseteq \Re_{\aleph_{j}}^{-1}(\hat{X})$, let $u \in \Re_{\aleph_{j}}^{-1}(\hat{X})$. Since $u \in \Re_{\aleph_{j}}^{-1}(\hat{X})$ implies $\aleph_{j}^{\square}(u) \sqsubseteq \hat{X}$. Again, let $v \in \aleph_{j}^{\Gamma}(u)$. Then $\aleph_{j}^{\Gamma}(v) \sqsubseteq \aleph_{j}^{\Pi}(u)$ implies $\aleph_{j}^{\Gamma}(v) \sqsubseteq \hat{X}$. Thus $v \in$ $\Re_{\aleph_{j}}^{-1}(\hat{X})$ and so $\aleph_{j}^{\Gamma}(u) \sqsubseteq \Re_{\aleph_{j}}^{-1}(\hat{X})$ implies $u \in \Re_{\aleph_{j}}^{-1}\left(\Re_{\aleph_{j}}^{-1}(\hat{X})\right)$. Therefore, (L9) is holding.

(K) Let $u \notin\left(\Re_{\aleph_{j}}^{-1}\left(\hat{X}_{1}\right)\right)^{c} \sqcup \Re_{\aleph_{j}}^{-1}\left(\hat{X}_{2}\right)$. Then $u \notin\left(\Re_{\aleph_{j}}^{-1}\left(\hat{X}_{1}\right)\right)^{c} \wedge$ $u \notin \Re_{\aleph_{j}}^{-1}\left(\hat{X}_{2}\right)$ and $u \in\left(\Re_{\aleph_{j}}^{-1}\left(\hat{X}_{1}\right)\right) \wedge u \notin \Re_{\aleph_{j}}^{-1}\left(\hat{X}_{2}\right)$, that implies $\aleph_{j}(u) \sqsubseteq \hat{X}_{1}$ and $\aleph_{j}(u) \nsubseteq \hat{X}_{2}$. Then $\aleph_{j}^{\Gamma}(u) \nsubseteq \hat{X}_{1}^{c}$ and $\aleph_{j}^{\sqcap}(u) \nsubseteq \hat{X}_{2}$. Hence, $\aleph_{j}^{\sqcap}(u) \nsubseteq \hat{X}_{1}^{c} \sqcup \hat{X}_{2}$ and $u \notin \Re_{\aleph_{j}}^{-1}\left(\hat{X}_{1}^{c} \sqcup \hat{X}_{2}\right)$. Therefore, $\Re_{\aleph_{j}}^{-1}\left(\hat{X}_{1}^{c} \sqcup \hat{X}_{2}\right) \sqsubseteq\left(\Re_{\aleph_{j}}^{-1}\left(\hat{X}_{1}\right)\right)^{c} \sqcup \Re_{\aleph_{j}}^{-1}\left(\hat{X}_{2}\right)$.

The equality of (L4), (U3), and (K) in Proposition 3.6 does not hold as shown in the following example.

Example 3.7. (Continued from Example 3.2). Take $j=j_{4}$ (and also $j \in\left\{j_{1}, j_{2}, j_{3}, j_{5}, j_{6}, j_{7}, j_{8}\right\}$ are similarly). Let $\hat{X}_{1}=$ $\{1,2\}$ and $\hat{X}_{2}=\{3\}$. Then $\Re_{\aleph_{j}}^{-1}\left(\hat{X}_{1}\right)=\{2\}, \Re_{\aleph_{j}}^{-1}\left(\hat{X}_{2}\right)=$ $\{3\}, \Re_{\aleph_{j}}^{+1}\left(\hat{X}_{1}\right)=\{1,2\}, \Re_{\aleph_{j}}^{+1}\left(\hat{X}_{2}\right)=\{1,3\}, \Re_{\aleph_{j}}^{-1}\left(\hat{X}_{1} \sqcup \hat{X}_{2}\right)=$ $\{1,2,3\}$ and $\Re_{\aleph_{j}}^{+1}\left(\hat{X}_{1} \sqcap \hat{X}_{2}\right)=\emptyset$. Then $\Re_{\aleph_{j}}^{-1}\left(\hat{X}_{1} \sqcup \hat{X}_{2}\right) \neq \Re_{\aleph_{j}}^{-1}\left(\hat{X}_{1}\right)$ $\sqcup \Re_{\aleph_{j}}^{-1}\left(\hat{X}_{2}\right)$ and $\Re_{\aleph_{j}}^{+1}\left(\hat{X}_{1} \sqcap \hat{X}_{2}\right) \neq \Re_{\aleph_{j}}^{+1}\left(\hat{X}_{1}\right) \sqcap \Re_{\aleph_{j}}^{+1}\left(\hat{X}_{2}\right)$. Let $\hat{X}_{1}=\{2,3\}$ and $\hat{X}_{2}=\{3,4\}$, then $\Re_{\aleph_{j}}^{-1}\left(\hat{X}_{1}^{c} \sqcup \hat{X}_{2}\right)=\{3,4\}$ and $\left(\Re_{\aleph_{j}}^{-1}\left(\hat{X}_{1}\right)\right)^{c} \sqcup \Re_{\aleph_{j}}^{-1}\left(\hat{X}_{2}\right)=\{1,3,4\}$. Thus $\Re_{\aleph_{j}}^{-1}\left(\hat{X}_{1}^{c} \sqcup \hat{X}_{2}\right) \neq$ $\left(\Re_{\aleph_{j}}^{-1}\left(\hat{X}_{1}\right)\right)^{c} \sqcup \Re_{\aleph_{j}}^{-1}\left(\hat{X}_{2}\right)$.

Definition 3.8. Let $\left(\mathcal{U}, \Re, \vartheta_{j}\right)$ be a $j$-RNS. A reflexive $2 j$ neighborhood rough set of $\hat{X} \sqsubseteq \mathcal{U}$ in $\left(\mathcal{U}, \Re, \vartheta_{j}\right)$ or with respect to $\vartheta_{j}$ is a pair $\left(\Re_{\aleph_{j}}^{-2}(\hat{X}), \Re_{\aleph_{j}}^{+2}(\overline{\hat{X}})\right)$, defined by

$$
\Re_{\aleph_{j}}^{-2}(\hat{X})=\bigsqcup\left\{\aleph_{j}^{\sqcap}(u): \aleph_{j}^{\sqcap}(u) \sqsubseteq \hat{X}\right\}
$$

(called the reflexive 2-lower approximation of $\hat{X}$ ) and

$$
\Re_{\aleph_{j}}^{+2}(\hat{X})=\left[\Re_{\aleph_{j}}^{-2}\left(\hat{X}^{c}\right)\right]^{c}
$$

(called the reflexive 2-upper approximation of $\hat{X}$ ).

Example 3.9 (Continued from Example 3.2). Take $j=j_{2}$ (and also $j \in\left\{j_{1}, j_{3}, j_{4}, j_{5}, j_{6}, j_{7}, j_{8}\right\}$ are similarly). Let $X=$ $\{1,3\}$. Then $\Re_{\aleph_{j}}^{-2}(\hat{X})=\{1,3\}, \Re_{\aleph_{j}}^{+2}(\hat{X})=\{1,2,3\}$.

Remark 3.10. When $j=j_{1}$ in Definition 3.8, then we obtain on Definition 2.5. 
Proposition 3.11. Let $\hat{X}, \hat{X}_{1}, \hat{X}_{2} \sqsubseteq \mathcal{U}$. Then the following properties (L1)-(L7), (L9), (U1)-(U7), (U9), (K), and (LU) will be satisfy based on Definition 3.8 .

Proof. The following properties (L2), (L4), (L6), (L7), (U1)(U7), (U9) and (LU) follows from Definition 3.8.

(L1) As $\left[\Re_{\aleph_{j}}^{+2}\left(\hat{X}^{c}\right)\right]^{c}=\left[\left[\left[\Re_{\aleph_{j}}^{-2}\left(\hat{X}^{c}\right)\right]^{c}\right]^{c}\right]^{c}=\Re_{\aleph_{j}}^{-2}(\hat{X})$.

(L3) As $\Re_{\aleph_{j}}^{-2}\left(\hat{X}_{1} \sqcap \hat{X}_{2}\right)=\bigsqcup\left\{\aleph_{j}^{\sqcap}(u): \aleph_{j}^{\sqcap}(u) \sqsubseteq \hat{X}_{1} \sqcap \hat{X}_{2}\right\}=$ $\bigsqcup\left\{\aleph_{j}(u): \aleph_{j}^{\Pi}(u) \sqsubseteq \hat{X}_{1} \wedge \aleph_{j}^{\Pi}(u) \sqsubseteq \hat{X}_{2}\right\}=\bigsqcup\left\{\aleph_{j}^{\Pi}(u): \aleph_{j}^{\Pi}(u) \sqsubseteq\right.$ $\left.\hat{X}_{1}\right\} \sqcap \bigsqcup\left\{\aleph_{j}^{\Gamma}(u): \aleph_{j}^{\sqcap}(u) \sqsubseteq \hat{X}_{2}\right\}=\Re_{\aleph_{j}}^{-2}\left(\hat{X}_{1}\right) \sqcap \Re_{\aleph_{j}}^{-2}\left(\hat{X}_{2}\right)$.

(L5) Let $\hat{X}_{1} \sqsubseteq \hat{X}_{2}$ and $u \in \Re_{\aleph_{j}}^{-2}\left(\hat{X}_{1}\right)$. Then $\aleph_{j}^{\sqcap}(u) \sqsubseteq \hat{X}_{1}$ and so $\aleph_{j}^{\sqcap}(u) \sqsubseteq \hat{X}_{2}$ implies $u \in \Re_{\aleph_{j}}^{-2}\left(\hat{X}_{2}\right)$. Thus $\Re_{\aleph_{j}}^{-2}\left(\hat{X}_{1}\right) \sqsubseteq$ $\Re_{\aleph_{j}}^{-2}\left(\hat{X}_{2}\right)$.

(L9) By (L7), we obtain $\Re_{\aleph_{j}}^{-2}\left(\Re_{\aleph_{j}}^{-2}(\hat{X})\right) \sqsubseteq \Re_{\aleph_{j}}^{-2}(\hat{X})$. Now, we show $\Re_{\aleph_{j}}^{-2}(\hat{X}) \sqsubseteq \Re_{\aleph_{j}}^{-2}\left(\Re_{\aleph_{j}}^{-2}(\hat{X})\right)$. As $\aleph_{j}^{\Pi}(u) \sqsubseteq \Re_{\aleph_{j}}^{-2}(\hat{X})$, let $u \in \Re_{\aleph_{j}}^{-2}(\hat{X})$. Since $u \in \Re_{\aleph_{j}}^{-2}(\hat{X})$ implies $\aleph_{j}^{\sqcap}(u) \sqsubseteq \hat{X}$. Again, let $v \in \aleph_{j}(u)$. Then $\aleph_{j}^{\Pi}(v) \sqsubseteq \aleph_{j}^{\Pi}(u)$ implies $\aleph_{j}^{\Pi}(v) \sqsubseteq \hat{X}$. Thus $v \in$ $\Re_{\aleph_{j}}^{-2}(\hat{X})$ and so $\aleph_{j}^{\Gamma}(u) \sqsubseteq \Re_{\aleph_{j}}^{-2}(\hat{X})$ implies $u \in \Re_{\aleph_{j}}^{-2}\left(\Re_{\aleph_{j}}^{-2}(\hat{X})\right)$. Therefore, (L9) is holding.

(K) Let $u \notin\left(\Re_{\aleph_{j}}^{-2}\left(\hat{X}_{1}\right)\right)^{c} \sqcup \Re_{\aleph_{j}}^{-2}\left(\hat{X}_{2}\right)$. Then $u \notin\left(\Re_{\aleph_{j}}^{-2}\left(\hat{X}_{1}\right)\right)^{c}$ $\wedge u \notin \Re_{\aleph_{j}}^{-2}\left(\hat{X}_{2}\right)$ and $u \in\left(\Re_{\aleph_{j}}^{-2}\left(\hat{X}_{1}\right)\right) \wedge u \notin \Re_{\aleph_{j}}^{-2}\left(\hat{X}_{2}\right)$, that implies $\aleph_{j}^{\Pi}(u) \sqsubseteq \hat{X}_{1}$ and $\aleph_{j}^{\square}(u) \nsubseteq \hat{X}_{2}$. Then $\aleph_{j}^{\Pi}(u) \nsubseteq \hat{X}_{1}^{c}$ and $\aleph_{j}^{\sqcap}(u) \nsubseteq \hat{X}_{2}$. Hence, $\aleph_{j}^{\sqcap}(u) \nsubseteq \hat{X}_{1}^{c} \sqcup \hat{X}_{2}$ and $u \notin \Re_{\aleph_{j}}^{-2}\left(\hat{X}_{1}^{c} \sqcup \hat{X}_{2}\right)$. Therefore, $\Re_{\aleph_{j}}^{-2}\left(\hat{X}_{1}^{c} \sqcup \hat{X}_{2}\right) \sqsubseteq\left(\Re_{\aleph_{j}}^{-2}\left(\hat{X}_{1}\right)\right)^{c} \sqcup \Re_{\aleph_{j}}^{-2}\left(\hat{X}_{2}\right)$.

The equality of (L4), (U3), and (K) in Proposition 3.11 does not hold as shown in the following example.

Example 3.12. (Continued from Example 3.2). Take $j=j_{4}$ (and also $j \in\left\{j_{1}, j_{2}, j_{3}, j_{5}, j_{6}, j_{7}, j_{8}\right\}$ are similarly). Let $\hat{X}_{1}=$ $\{1,2\}$ and $\hat{X}_{2}=\{3\}$. Then $\Re_{\aleph_{j}}^{-2}\left(\hat{X}_{1}\right)=\{2\}, \Re_{\aleph_{j}}^{-2}\left(\hat{X}_{2}\right)=$ $\{3\}$ and $\Re_{\aleph_{j}}^{-2}\left(\hat{X}_{1} \sqcup \hat{X}_{2}\right)=\{1,2,3\}$ Then $\Re_{\aleph_{j}}^{-2}\left(\hat{X}_{1} \sqcup \hat{X}_{2}\right) \neq$ $\Re_{\aleph_{j}}^{-2}\left(\hat{X}_{1}\right) \sqcup \Re_{\aleph_{j}}^{-2}\left(\hat{X}_{2}\right)$. Let $\hat{X}_{1}=\{1,2\}$ and $\hat{X}_{2}=\{3,4\}$. Then $\Re_{\aleph_{j}}^{+2}\left(\hat{X}_{1}\right)=\{1,2\}, \Re_{\aleph_{j}}^{+2}\left(\hat{X}_{2}\right)=\{1,3,4\}$ and $\Re_{\aleph_{j}}^{+2}\left(\hat{X}_{1} \sqcap \hat{X}_{2}\right)=$ $\emptyset$. Thus $\Re_{\aleph_{j}}^{+2}\left(\hat{X}_{1} \sqcap \hat{X}_{2}\right) \neq \Re_{\aleph_{j}}^{+2}\left(\hat{X}_{1}\right) \sqcap \Re_{\aleph_{j}}^{+2}\left(\hat{X}_{2}\right)$. Let $\hat{X}_{1}=$ $\{2,3\}$ and $\hat{X}_{2}=\{3,4\}$, then $\Re_{\aleph_{j}}^{-2}\left(\hat{X}_{1}^{c} \sqcup \hat{X}_{2}\right)=\{3,4\}$ and $\left(\Re_{\aleph_{j}}^{-2}\left(\hat{X}_{1}\right)\right)^{c} \sqcup \Re_{\aleph_{j}}^{-2}\left(\hat{X}_{2}\right)=\{1,3,4\}$. Thus $\Re_{\aleph_{j}}^{-2}\left(\hat{X}_{1}^{c} \sqcup \hat{X}_{2}\right) \neq$ $\left(\Re_{\aleph_{j}}^{-2}\left(\hat{X}_{1}\right)\right)^{c} \sqcup \Re_{\aleph_{j}}^{-2}\left(\hat{X}_{2}\right)$.

Definition 3.13. Let $\left(\mathcal{U}, \Re, \vartheta_{j}\right)$ be a $j$-RNS. A reflexive $3 j$ neighborhood rough set of $\hat{X} \sqsubseteq \mathcal{U}$ in $\left(\mathcal{U}, \Re, \vartheta_{j}\right)$ or with respect to $\vartheta_{j}$ is a pair $\left(\Re_{\aleph_{j}}^{-3}(\hat{X}), \Re_{\aleph_{j}}^{+3}(\overline{\hat{X}})\right)$, defined by

$$
\Re_{\aleph_{j}}^{-3}(\hat{X})=\left[\Re_{\aleph_{j}}^{+3}\left(\hat{X}^{c}\right)\right]^{c}
$$

(called the reflexive 3-lower approximation of $\hat{X}$ ) and

$$
\Re_{\aleph_{j}}^{+3}(\hat{X})=\bigsqcup\left\{\aleph_{j}^{\sqcap}(u): \aleph_{j}^{\sqcap}(u) \sqcap \hat{X} \neq \emptyset\right\}
$$

(called the reflexive 3-upper approximation of $\hat{X}$ ).

Example 3.14 (Continued from Example 3.2). Take $j=j_{2}$ (and also $j \in\left\{j_{1}, j_{3}, j_{4}, j_{5}, j_{6}, j_{7}, j_{8}\right\}$ are similarly). Let $X=$ $\{1,3\}$. Then $\Re_{\aleph_{j}}^{-3}(\hat{X})=\{3\}, \Re_{\aleph_{j}}^{+3}(\hat{X})=\{1,2,3\}$.
Remark 3.15. When $j=j_{1}$ in Definition 3.13, then we obtain on Definition 2.6.

Proposition 3.16. LLet $\hat{X}, \hat{X}_{1}, \hat{X}_{2} \sqsubseteq \mathcal{U}$. Then the following properties (L1)-(L8), (U1)-(U8), (K), and (LU) will be satisfy based on Definition 3.13.

Proof. The following properties (L1)-(L8), (U2), (U4), (U6), (U7) and (LU) follows from Definition 3.13.

(U1) As $\left[\Re_{\aleph_{j}}^{-3}\left(\hat{X}^{c}\right)\right]^{c}=\left[\left[\left[\Re_{\aleph_{j}}^{+3}\left(\hat{X}^{c}\right)\right]^{c}\right]^{c}\right]^{c}=\Re_{\aleph_{j}}^{+3}(\hat{X})$.

(U3) As $\Re_{\aleph_{j}}^{+3}\left(\hat{X}_{1} \sqcup \hat{X}_{2}\right)=\bigsqcup\left\{\aleph_{j}^{\square}(u): \aleph_{j}^{\sqcap}(u) \sqcap\left(\hat{X}_{1} \sqcup \hat{X}_{2}\right) \neq\right.$ $\emptyset\}=\bigsqcup\left\{\aleph_{j}^{\Pi}(u): \aleph_{j}^{\Pi}(u) \sqcap \hat{X}_{1} \neq \emptyset \vee \aleph_{j}^{\Pi}(u) \sqcap \hat{X}_{2} \neq \emptyset\right\}=$ $\bigsqcup\left\{\aleph_{j}(u): \aleph_{j}^{\Gamma_{j}}(u) \sqcap \hat{X}_{1} \neq \emptyset\right\} \sqcup \sqcup\left\{\aleph_{j}(u): \aleph_{j}^{\Gamma_{j}}(u) \sqcap \hat{X}_{2} \neq \emptyset\right\}=$ $\Re_{\aleph_{j}}^{+3}\left(\hat{X}_{1}\right) \sqcup \Re_{\aleph_{j}}^{+3}\left(\hat{X}_{2}\right)$.

(U5) Let $\hat{X}_{1} \sqsubseteq \hat{X}_{2}$ and $u \in \Re_{\aleph_{j}}^{+3}\left(\hat{X}_{1}\right)$. Then $\aleph_{j}^{\square}(u) \sqsubseteq \hat{X}_{1}$ and so $\aleph_{j}^{\Pi}(u) \sqsubseteq \hat{X}_{2}$ implies $u \in \Re_{\aleph_{j}}^{+3}\left(\hat{X}_{2}\right)$. Thus $\Re_{\aleph_{j}}^{+3}\left(\hat{X}_{1}\right) \sqsubseteq$ $\Re_{\aleph_{j}}^{+3}\left(\hat{X}_{2}\right)$.

(U8) Let $u \in \Re_{\aleph_{j}}^{+3}\left(\Re_{\aleph_{j}}^{-3}(\hat{X})\right)$. Then there exist $v \in \mathcal{U}$ such that $v \in \aleph_{j}^{\Pi}(u)$ and $\aleph_{j}^{\Pi}(u) \sqcap \Re_{\aleph_{j}}^{-3}(\hat{X}) \neq \emptyset$. Hence, there exist $w \in$ $\mathcal{U}$ such that $w \in \aleph_{j}^{\Pi}(u)$ and $w \in \Re_{\aleph_{j}}^{-3}(\hat{X})$, thus $w \notin \Re_{\aleph_{j}}^{+3}\left(\hat{X}^{c}\right)$ and $\aleph_{j}(u) \sqcap \hat{X}^{c} \neq \emptyset$, then $\aleph_{j}^{\sqcap}(u) \sqsubseteq \hat{X}$, hence $w \in \hat{X}$. Therefore $\Re_{\aleph_{j}}^{+3}\left(\Re_{\aleph_{j}}^{-3}(\hat{X})\right) \sqsubseteq(\hat{X})$.

(K) Let $u \notin\left(\Re_{\aleph_{j}}^{-3}\left(\hat{X}_{1}\right)\right)^{c} \sqcup \Re_{\aleph_{j}}^{-3}\left(\hat{X}_{2}\right)$. Then $u \notin\left(\Re_{\aleph_{j}}^{-3}\left(\hat{X}_{1}\right)\right)^{c}$ $\wedge u \notin \Re_{\aleph_{j}}^{-3}\left(\hat{X}_{2}\right)$ and $u \in\left(\Re_{\aleph_{j}}^{-3}\left(\hat{X}_{1}\right)\right) \wedge u \notin \Re_{\aleph_{j}}^{-3}\left(\hat{X}_{2}\right)$, that implies $\aleph_{j}^{\Gamma}(u) \sqsubseteq \hat{X}_{1}$ and $\aleph_{j}^{\Pi}(u) \nsubseteq \hat{X}_{2}$. Then $\aleph_{j}^{\Pi}(u) \nsubseteq \hat{X}_{1}^{c}$ and $\aleph_{j}^{\Gamma}(u) \nsubseteq \hat{X}_{2}$. Hence, $\aleph_{j}^{\sqcap}(u) \nsubseteq \hat{X}_{1}^{c} \sqcup \hat{X}_{2}$ and $u \notin \Re_{\aleph_{j}}^{-3}\left(\hat{X}_{1}^{c} \sqcup \hat{X}_{2}\right)$. Therefore, $\Re_{\aleph_{j}}^{-3}\left(\hat{X}_{1}^{c} \sqcup \hat{X}_{2}\right) \sqsubseteq\left(\Re_{\aleph_{j}}^{-3}\left(\hat{X}_{1}\right)\right)^{c} \sqcup \Re_{\aleph_{j}}^{-3}\left(\hat{X}_{2}\right)$.

The equality of (L4), (L8), (U3), (U8) and (K) in Proposition 3.16 does not hold as shown in the following example.

Example 3.17. (Continued from Example 3.2). Take $j=j_{4}$ (and also $j \in\left\{j_{1}, j_{2}, j_{3}, j_{5}, j_{6}, j_{7}, j_{8}\right\}$ are similarly). Let $\hat{X}_{1}=$ $\{1,2\}$ and $\hat{X}_{2}=\{1,3\}$. Then $\Re_{\aleph_{j}}^{-3}\left(\hat{X}_{1}\right)=\Re_{\aleph_{j}}^{-3}\left(\hat{X}_{2}\right)=\emptyset$ and $\Re_{\aleph_{j}}^{-3}\left(\hat{X}_{1} \sqcup \hat{X}_{2}\right)=\{1,2,3\}$ Then $\Re_{\aleph_{j}}^{-3}\left(\hat{X}_{1} \sqcup \hat{X}_{2}\right) \neq \Re_{\aleph_{j}}^{-3}\left(\hat{X}_{1}\right) \sqcup$ $\Re_{\aleph_{j}}^{-3}\left(\hat{X}_{2}\right)$. Let $\hat{X}_{1}=\{1,2\}$ and $\hat{X}_{2}=\{3,4\}$. Then $\Re_{\aleph_{j}}^{+3}\left(\hat{X}_{1}\right)=$ $\{1,2,3\}, \Re_{\aleph_{j}}^{+3}\left(\hat{X}_{2}\right)=\mathcal{U}$ and $\Re_{\aleph_{j}}^{+3}\left(\hat{X}_{1} \sqcap \hat{X}_{2}\right)=\emptyset$. Thus $\Re_{\aleph_{j}}^{+3}\left(\hat{X}_{1} \sqcap\right.$ $\left.\hat{X}_{2}\right) \neq \Re_{\aleph_{j}}^{+3}\left(\hat{X}_{1}\right) \sqcap \Re_{\aleph_{j}}^{+3}\left(\hat{X}_{2}\right) . \Re_{\aleph_{j}}^{-3}\left(\Re_{\aleph_{j}}^{+3}\left(\hat{X}_{1}\right)\right)=\{1,2,3\}$ and $\Re_{\aleph_{j}}^{+3}\left(\Re_{\aleph_{j}}^{-3}\left(\hat{X}_{2}\right)\right)=\emptyset$, then $\hat{X}_{1} \neq \Re_{\aleph_{j}}^{-3}\left(\Re_{\aleph_{j}}^{+3}\left(\hat{X}_{1}\right)\right)$ and $\hat{X}_{2} \neq$ $\Re_{\aleph_{j}}^{+3}\left(\Re_{\aleph_{j}}^{-3}\left(\hat{X}_{2}\right)\right)$. Let $\hat{X}_{1}=\{2,3\}$ and $\hat{X}_{2}=\{3,4\}$, then $\Re_{\aleph_{j}}^{-3}\left(\hat{X}_{1}^{c} \sqcup\right.$ $\left.\hat{X}_{2}\right)=\{4\}$ and $\left(\Re_{\aleph_{j}}^{-3}\left(\hat{X}_{1}\right)\right)^{c} \sqcup \Re_{\aleph_{j}}^{-3}\left(\hat{X}_{2}\right)=\mathcal{U}$. Thus $\Re_{\aleph_{j}}^{-3}\left(\hat{X}_{1}^{c} \sqcup\right.$ $\left.\hat{X}_{2}\right) \neq\left(\Re_{\aleph_{j}}^{-3}\left(\hat{X}_{1}\right)\right)^{c} \sqcup \Re_{\aleph_{j}}^{-3}\left(\hat{X}_{2}\right)$.

\subsection{Second kind: Generalized rough set models by Dai et al.'s approach [25] based on j-similarity neighborhood space}

In this subsection, we will extend the Definitions 2.7, 2.8, 2.9 and 2.10 to $j$-similarity neighborhood space as indicated below.

Definition 3.18. Let $\left(\mathcal{U}, \Re, \vartheta_{j}\right)$ be a $j$-neighborhood space, we define a set $\aleph_{j}(u)(\forall u \in U)$ (The union of all $j$-neighborhoods containing $u$ ) as follows:

$$
\aleph_{j}^{\sqcup}(u)=\left\{\begin{array}{ll}
\bigsqcup_{u \in \aleph_{j}(v)} \aleph_{j}(v), & \text { if } \exists v, u \in \aleph_{j}(v) \\
\emptyset, & \text { otherwise }
\end{array} .\right.
$$


Example 3.19. Let $\mathcal{U}=\{1,2,3,4\}$ and $\Re \sqsubseteq \mathcal{U} \times \mathcal{U}$ be a similarity relation on $\mathcal{U}$ defined by

$\Re=\{(1,1),(1,2),(1,4),(2,1),(2,2),(2,3),(3,3),(3,2)$, $(4,1),(4,4)\}$. Then the $\aleph_{j}(u)\left(\forall u \in U, \forall j \in\left\{j_{1}, j_{2}, j_{3}, j_{4}, j_{5}\right.\right.$, $\left.\left.j_{6}, j_{7}, j_{8}\right\}\right)$ is

$$
\begin{gathered}
\aleph_{j}(1)=\{1,2,4\}, \aleph_{j}(2)=\{1,2,3\}, \\
\aleph_{j}(3)=\{2,3\}, \aleph_{j}(4)=\{1,4\}
\end{gathered}
$$

and the $\aleph_{j}^{\sqcup}(u)\left(\forall u \in U, \forall j \in\left\{j_{1}, j_{2}, j_{3}, j_{4}, j_{5}, j_{6}, j_{7}, j_{8}\right\}\right)$ is

$$
\begin{gathered}
\aleph_{j}^{\sqcup}(1)=\mathcal{U}, \quad \aleph_{j}^{\sqcup}(2)=\mathcal{U} \\
\aleph_{j}^{\sqcup}(3)=\{1,2,3\}, \quad \aleph_{j}^{\sqcup}(4)=\{1,2,4\} .
\end{gathered}
$$

Definition 3.20. Let $\left(\mathcal{U}, \Re, \vartheta_{j}\right)$ be a $j$-SNS. A similarity $4 j$ neighborhood rough set of $\hat{X} \sqsubseteq \mathcal{U}$ in $\left(\mathcal{U}, \Re, \vartheta_{j}\right)$ or with respect to $\vartheta_{j}$ is a pair $\left(\Re_{\aleph_{j}}^{-4}(\hat{X}), \Re_{\aleph_{j}}^{+4}(\hat{X})\right)$, defined by

$$
\Re_{\aleph_{j}}^{-4}(\hat{X})=\left\{u \in \mathcal{U}: \aleph_{j}^{\sqcup}(u) \sqsubseteq \hat{X}\right\}
$$

(called the similarity 4-lower approximation of $\hat{X}$ )

and

$$
\Re_{\aleph_{j}}^{+4}(\hat{X})=\left\{u \in \mathcal{U}: \aleph_{j}^{\sqcup}(u) \sqcap \hat{X} \neq \emptyset\right\}
$$

(called the similarity 4-upper approximation of $\hat{X}$ ).

Example 3.21 (Continued from Example 3.19). Let $\hat{X}=$ $\{1,3\}$ and take $\forall j \in\left\{j_{1}, j_{2}, j_{3}, j_{4}, j_{5}, j_{6}, j_{7}, j_{8}\right\}$. Then

$$
\Re_{\aleph_{j}}^{-4}(\hat{X})=\emptyset \text { and } \Re_{\aleph_{j}}^{+4}(\hat{X})=\mathcal{U}
$$

Remark 3.22. If we consider $j=j_{1}$ in Definition 3.20, then we obtain on Definition 2.8.

Proposition 3.23. Let $\hat{X}, \hat{X}_{1}, \hat{X}_{2} \sqsubseteq \mathcal{U}$. Then the following properties (L1)-(L8), (U1)-(U8), (K), and (LU) will be satisfy based on Definition 3.20.

Proof. Similar to Proposition 3.8.

The equality of (L4), (U3), and (K) in Proposition 3.23 does not hold as shown in the following example.

Example 3.24. (Continued from Example 3.19). Take $j=j_{3}$ (and also $j \in\left\{j_{1}, j_{2}, j_{4}, j_{5}, j_{6}, j_{7}, j_{8}\right\}$ are similarly). Let $\hat{X}_{1}=$ $\{1,2\}$ and $\hat{X}_{2}=\{3\}$. Then $\Re_{\aleph_{j}}^{-4}\left(\hat{X}_{1}\right)=\emptyset, \Re_{\aleph_{j}}^{-4}\left(\hat{X}_{2}\right)=\emptyset$, $\Re_{\aleph_{j}}^{+4}\left(\hat{X}_{1}\right)=\mathcal{U}, \Re_{\aleph_{j}}^{+4}\left(\hat{X}_{2}\right)=\{1,2,3\}, \Re_{\aleph_{j}}^{-4}\left(\hat{X}_{1} \sqcup \hat{X}_{2}\right)=\{3\}$ and $\Re_{\aleph_{j}}^{+4}\left(\hat{X}_{1} \sqcap \hat{X}_{2}\right)=\emptyset$. Then $\Re_{\aleph_{j}}^{-4}\left(\hat{X}_{1} \sqcup \hat{X}_{2}\right) \neq \Re_{\aleph_{j}}^{-4}\left(\hat{X}_{1}\right) \sqcup \Re_{\aleph_{j}}^{-4}\left(\hat{X}_{2}\right)$ and $\Re_{\aleph_{j}}^{+4}\left(\hat{X}_{1} \sqcap \hat{X}_{2}\right) \neq \Re_{\aleph_{j}}^{+4}\left(\hat{X}_{1}\right) \sqcap \Re_{\aleph_{j}}^{+4}\left(\hat{X}_{2}\right)$. Let $\hat{X}_{1}=\{2,3\}$ and $\hat{X}_{2}=\{3,4\}$, then $\Re_{\aleph_{j}}^{-4}\left(\hat{X}_{1}^{c} \sqcup \hat{X}_{2}\right)=\emptyset$ and $\left(\Re_{\aleph_{j}}^{-4}\left(\hat{X}_{1}\right)\right)^{c} \sqcup$ $\Re_{\aleph_{j}}^{-4}\left(\hat{X}_{2}\right)=\mathcal{U}$. Thus $\Re_{\aleph_{j}}^{-4}\left(\hat{X}_{1}^{c} \sqcup \hat{X}_{2}\right) \neq\left(\Re_{\aleph_{j}}^{-4}\left(\hat{X}_{1}\right)\right)^{c} \sqcup \Re_{\aleph_{j}}^{-4}\left(\hat{X}_{2}\right)$.

Definition 3.25. Let $\left(\mathcal{U}, \Re, \vartheta_{j}\right)$ be a $j$-SNS. A similarity $5 j$ neighborhood rough set of $\hat{X} \sqsubseteq \mathcal{U}$ in $\left(\mathcal{U}, \Re, \vartheta_{j}\right)$ or with respect to $\vartheta_{j}$ is a pair $\left(\Re_{\aleph_{j}}^{-5}(\hat{X}), \Re_{\aleph_{j}}^{+5}(\hat{X})\right)$, defined by

$$
\Re_{\aleph_{j}}^{-5}(\hat{X})=\bigsqcup\left\{\aleph_{j}^{\sqcup}(u): \aleph_{j}^{\sqcup}(u) \sqsubseteq \hat{X}\right\}
$$

(called the similarity 5-lower approximation of $\hat{X}$ ) and

$$
\Re_{\aleph_{j}}^{+5}(\hat{X})=\left[\Re_{\aleph_{j}}^{-5}\left(\hat{X}^{c}\right)\right]^{c}
$$

(called the similarity 5-upper approximation of $\hat{X}$ ).
Example 3.26 (Continued from Example 3.19). Take $j=j_{3}$ (and also $j \in\left\{j_{1}, j_{2}, j_{4}, j_{5}, j_{6}, j_{7}, j_{8}\right\}$ are similarly). Let $X=$ $\{1,3\}$. Then $\Re_{\aleph_{j}}^{-5}(\hat{X})=\emptyset, \Re_{\aleph_{j}}^{+5}(\hat{X})=\mathcal{U}$.

Remark 3.27. If we consider $j=j_{1}$ in Definition 3.25, then we obtain on Definition 2.9.

Proposition 3.28. Let $\hat{X}, \hat{X}_{1}, \hat{X}_{2} \sqsubseteq \mathcal{U}$. Then the following properties (L1)-(L2), (L4)-(L7), (L9) (U1)-(U2), (U4)-(U7), (U9) (K), and (LU) will be satisfy based on Definition 3.25.

Proof. Similar to Proposition 3.11.

The equality of (L4) and (K) in Proposition 3.28 does not hold as shown in the following example.

Example 3.29 (Continued from Example 3.19). Take $j=j_{3}$ (and also $j \in\left\{j_{1}, j_{2}, j_{4}, j_{5}, j_{6}, j_{7}, j_{8}\right\}$ are similarly). Let $\hat{X}_{1}=$ $\{1,2\}$ and $\hat{X}_{2}=\{3\}$. Then $\Re_{\aleph_{j}}^{-5}\left(\hat{X}_{1}\right)=\emptyset, \Re_{\aleph_{j}}^{-5}\left(\hat{X}_{2}\right)=\emptyset$ and $\Re_{\aleph_{j}}^{-5}\left(\hat{X}_{1} \sqcup \hat{X}_{2}\right)=\{1,2,3\}$. Then $\Re_{\aleph_{j}}^{-5}\left(\hat{X}_{1} \sqcup \hat{X}_{2}\right) \neq \Re_{\aleph_{j}}^{-5}\left(\hat{X}_{1}\right)$ $\sqcup \Re_{\aleph_{j}}^{-5}\left(\hat{X}_{2}\right)$. Let $\hat{X}_{1}=\{2,3\}$ and $\hat{X}_{2}=\{3,4\}$, then $\Re_{\aleph_{j}}^{-5}\left(\hat{X}_{1}^{c} \sqcup\right.$ $\left.\hat{X}_{2}\right)=\emptyset$ and $\left(\Re_{\aleph_{j}}^{-5}\left(\hat{X}_{1}\right)\right)^{c} \sqcup \Re_{\aleph_{j}}^{-5}\left(\hat{X}_{2}\right)=\mathcal{U}$. Thus $\Re_{\aleph_{j}}^{-5}\left(\hat{X}_{1}^{c} \sqcup\right.$ $\left.\hat{X}_{2}\right) \neq\left(\Re_{\aleph_{j}}^{-5}\left(\hat{X}_{1}\right)\right)^{c} \sqcup \Re_{\aleph_{j}}^{-5}\left(\hat{X}_{2}\right)$.

Definition 3.30. Let $\left(\mathcal{U}, \Re, \vartheta_{j}\right)$ be a $j$-SNS. A similarity $6 j$ neighborhood rough set of $\hat{X} \sqsubseteq \mathcal{U}$ in $\left(\mathcal{U}, \Re, \vartheta_{j}\right)$ or with respect to $\vartheta_{j}$ is a pair $\left(\Re_{\aleph_{j}}^{-6}(\hat{X}), \Re_{\aleph_{j}}^{+6}(\hat{X})\right)$, defined by

$$
\Re_{\aleph_{j}}^{-6}(\hat{X})=\left[\Re_{\aleph_{j}}^{+6}\left(\hat{X}^{c}\right)\right]^{c}
$$

(called the similarity 6-lower approximation of $\hat{X}$ ) and

$$
\Re_{\aleph_{j}}^{+6}(\hat{X})=\bigsqcup\left\{\aleph_{j}^{\sqcup}(u): \aleph_{j}^{\sqcup}(u) \sqcap \hat{X} \neq \emptyset\right\}
$$

(called the similarity 6-upper approximation of $\hat{X}$ ).

Example 3.31 (Continued from Example 3.19). Take $j=j_{2}$ (and also $j \in\left\{j_{1}, j_{3}, j_{4}, j_{5}, j_{6}, j_{7}, j_{8}\right\}$ are similarly). Let $X=$ $\{1,3\}$. Then $\Re_{\aleph_{j}}^{-6}(\hat{X})=\emptyset, \Re_{\aleph_{j}}^{+6}(\hat{X})=\mathcal{U}$.

Remark 3.32. When $j=j_{1}$ in Definition 3.30, then we obtain on Definition 2.10.

Proposition 3.33. Let $\hat{X}, \hat{X}_{1}, \hat{X}_{2} \sqsubseteq \mathcal{U}$. Then the following properties (L1)-(L8), (U1)-(U8), (K), and (LU) will be satisfy based on Definition 3.30.

Proof. Similar to Proposition 3.16 .

The equality of (L4), (L8), (U3), (U8) and (K) in Proposition 3.33 does not hold as shown in the following example.

Example 3.34 (Continued from Example 3.19). Take $j=j_{3}$ (and also $j \in\left\{j_{1}, j_{2}, j_{4}, j_{5}, j_{6}, j_{7}, j_{8}\right\}$ are similarly). Let $\hat{X}_{1}=$ $\{1,2\}$ and $\hat{X}_{2}=\{3,4\}$. Then $\Re_{\aleph_{j}}^{-6}\left(\hat{X}_{1}\right)=\Re_{\aleph_{j}}^{-6}\left(\hat{X}_{2}\right)=\emptyset$ and $\Re_{\aleph_{j}}^{-6}\left(\hat{X}_{1} \sqcup \hat{X}_{2}\right)=\mathcal{U}$ Then $\Re_{\aleph_{j}}^{-6}\left(\hat{X}_{1} \sqcup \hat{X}_{2}\right) \neq \Re_{\aleph_{j}}^{-6}\left(\hat{X}_{1}\right) \sqcup \Re_{\aleph_{j}}^{-6}\left(\hat{X}_{2}\right)$. Let $\hat{X}_{1}=\{1,2\}$ and $\hat{X}_{2}=\{3,4\}$. Then $\Re_{\aleph_{j}^{+6}}^{+6}\left(\hat{X}_{1}\right)=\mathcal{U}, \Re_{\aleph_{j}}^{+6}\left(\hat{X}_{2}\right)=$ $\mathcal{U}$ and $\Re_{\aleph_{j}}^{+6}\left(\hat{X}_{1} \sqcap \hat{X}_{2}\right)=\emptyset$. Thus $\Re_{\aleph_{j}}^{+6}\left(\hat{X}_{1} \sqcap \hat{X}_{2}\right) \neq \Re_{\aleph_{j}}^{+6}\left(\hat{X}_{1}\right) \sqcap$ $\Re_{\aleph_{j}}^{+6}\left(\hat{X}_{2}\right) . \Re_{\aleph_{j}}^{-6}\left(\Re_{\aleph_{j}}^{+6}\left(\hat{X}_{1}\right)\right)=\mathcal{U}$ and $\Re_{\aleph_{j}}^{+6}\left(\Re_{\aleph_{j}}^{-6}\left(\hat{X}_{2}\right)\right)=\emptyset$, then $\hat{X}_{1} \neq \Re_{\aleph_{j}}^{-6}\left(\Re_{\aleph_{j}}^{+6}\left(\hat{X}_{1}\right)\right)$ and $\hat{X}_{2} \neq \Re_{\aleph_{j}}^{+6}\left(\Re_{\aleph_{j}}^{-6}\left(\hat{X}_{2}\right)\right)$. Let $\hat{X}_{1}=$ $\{2,3\}$ and $\hat{X}_{2}=\{3,4\}$, then $\Re_{\aleph_{j}}^{-6}\left(\hat{X}_{1}^{c} \sqcup \hat{X}_{2}\right)=\emptyset$ and $\left(\Re_{\aleph_{j}}^{-6}\left(\hat{X}_{1}\right)\right)^{c} \sqcup$ $\Re_{\aleph_{j}}^{-6}\left(\hat{X}_{2}\right)=\mathcal{U}$. Thus $\Re_{\aleph_{j}}^{-6}\left(\hat{X}_{1}^{c} \sqcup \hat{X}_{2}\right) \neq\left(\Re_{\aleph_{j}}^{-6}\left(\hat{X}_{1}\right)\right)^{c} \sqcup \Re_{\aleph_{j}}^{-6}\left(\hat{X}_{2}\right)$. 


\section{New generalized rough set models based on j-adhesion neighborhood space}

In this section, we will extent Section 3 to $j$-adhesion neighborhood space as indicated below.

\subsection{Third kind: Generalized rough set models by based on} j-reflexive adhesion neighborhood space

Definition 4.1. Let $\Re$ be reflexive on $\mathcal{U}$. The $j$-reflexive adhesion neighborhood of element $u \in \mathcal{U}$ are defined as follows: $\forall j \in\left\{j_{1}, j_{2}, j_{3}, j_{4}, j_{5}, j_{6}, j_{7}, j_{8}\right\}$

(1) $\hbar_{j_{1}}(u)=\left\{v \in \mathcal{U}: \aleph_{j_{1}}^{\Pi_{1}}(u)=\aleph_{j_{1}}^{\Gamma_{1}}(v)\right\}$.

(2) $\hbar_{j_{2}}(u)=\left\{v \in \mathcal{U}: \aleph_{j_{2}}^{\Gamma}(u)=\aleph_{j_{2}}^{\Gamma_{1}}(v)\right\}$.

(3) $\hbar_{j_{3}}(u)=\left\{v \in \mathcal{U}: \bigcap_{u \in \aleph_{j_{1}}(v)} \aleph_{j_{1}}^{\Gamma_{1}}(v)=\bigcap_{v \in \aleph_{j_{1}}(x)} \aleph_{j_{1}}^{\Gamma_{1}}(u)\right\}$.

(4) $\hbar_{j_{4}}(u)=\left\{v \in \mathcal{U}: \bigcap_{u \in \aleph_{j_{2}}(v)} \aleph_{j_{2}}^{\Gamma_{2}}(v)=\bigcap_{v \in \aleph_{j_{2}}(u)}^{\Pi} \aleph_{j_{2}}^{\Pi}(u)\right\}$.

(5) $\hbar_{j_{5}}(u)=\hbar_{j_{1}}(u) \sqcup \hbar_{j_{2}}(u)$.

(6) $\hbar_{j_{6}}(u)=\hbar_{j_{1}}(u) \sqcap \hbar_{j_{2}}(u)$.

(7) $\hbar_{j_{7}}(u)=\hbar_{j_{3}}(u) \sqcup \hbar_{j_{4}}(u)$.

(8) $\hbar_{j_{8}}(u)=\hbar_{j_{3}}(u) \sqcap \hbar_{j_{4}}(u)$.

According to above Definition 4.1, we call $\left(\mathcal{U}, \Re, \vartheta_{j}\right)$ be a $j$ reflexive adhesion neighborhood space (briefly, a $j$ - $\mathbb{R} \mathbb{N} \mathbb{S}$ ).

Example 4.2 (Continued from Example 3.2). Take $j \in\left\{j_{1}, j_{2}\right.$, $\left.j_{3}, j_{4}, j_{5}, j_{6}, j_{7}, j_{8}\right\}$, Then the $j$-reflexive adhesion neighborhood is

$$
\begin{aligned}
& \hbar_{j}(1)=\{1\}, \quad \hbar_{j}(2)=\{2\}, \\
& \hbar_{j}(3)=\{3\}, \quad \hbar_{j}(4)=\{4\} .
\end{aligned}
$$

Definition 4.3. Let $\left(\mathcal{U}, \Re, \vartheta_{j}\right)$ be a $j-\mathbb{R} \mathbb{N} \mathbb{S}$. A reflexive $7 j$ adhesion neighborhood rough set of $\hat{X} \sqsubseteq \mathcal{U}$ in $\left(\mathcal{U}, \Re, \vartheta_{j}\right)$ or with respect to $\vartheta_{j}$ is a pair $\left(\Re_{\aleph_{j}}^{-7}(\hat{X}), \Re_{\aleph_{j}}^{+7}(\hat{X})\right)$, defined by

$$
\Re_{\aleph_{j}}^{-7}(\hat{X})=\left\{u \in \mathcal{U}: \hbar_{j}^{\Gamma}(u) \sqsubseteq \hat{X}\right\}
$$

(called the reflexive 7-lower approximation of $\hat{X}$ ) and

$$
\Re_{\aleph_{j}}^{+7}(\hat{X})=\left\{u \in \mathcal{U}: \hbar_{j}^{\sqcap}(u) \sqcap \hat{X} \neq \emptyset\right\}
$$

(called the reflexive 7-upper approximation of $\hat{X}$ ).

Example 4.4 (Continued from Example 4.2). Let $\hat{X}=\{1,3\}$ and take $j \in\left\{j_{1}, j_{2}, j_{3}, j_{4}, j_{5}, j_{6}, j_{7}, j_{8}\right\}$. Then

$$
\Re_{\aleph_{j}}^{-7}(\hat{X})=\{1,3\} \text { and } \Re_{\aleph_{j}^{+7}}^{+7}(\hat{X})=\{1,3\} \text {. }
$$

Proposition 4.5. Let $\hat{X}, \hat{X}_{1}, \hat{X}_{2} \sqsubseteq \mathcal{U}$. Then the following properties (L1)-(L10), (U1)-(U10), (K), and (LU) will be satisfy based on Definition 4.3.

Proof. We only prove (L9), (L10), (U9), and (U10).

(L9) Let $u \in \hat{X}$, for $v \in \hbar_{j}^{\Gamma}(u)$, then we have $u \in \hbar_{j}^{\Gamma}(v)$. Hence, $\hat{X} \cap \hbar_{j}^{\Gamma}(u) \neq \emptyset$. It follows that $v \in \Re_{\aleph_{j}}^{+7}(\hat{X})$. Thus, $\hbar_{j}^{\Gamma}(u) \subseteq \Re_{\aleph_{j}}^{+7}(\hat{X})$ and $u \in \Re_{\aleph_{j}}^{-7}\left(\Re_{\hbar_{j}}^{+7}(\hat{X})\right)$.

(L10) From (L7) and (L9).

(U9) Let $u \in \Re_{\aleph_{j}}^{+7}\left(\Re_{\aleph_{j}}^{-7}(\hat{X})\right)$. Then there exist $v \in \mathcal{U}$ such that $v \in \hbar_{j}^{\Gamma}(u)$ and $\hbar_{j}^{\Gamma}(u) \sqcap \Re_{\aleph_{j}}^{-7}(\hat{X}) \neq \emptyset$. Hence, there exist $w \in$ $\mathcal{U}$ such that $w \in \hbar_{j}^{\Gamma}(u)$ and $w \in \Re_{\aleph_{j}}^{-7}(\hat{X})$, thus $w \notin \Re_{\aleph_{j}}^{+7}\left(\hat{X}^{c}\right)$ and $\hbar_{j}^{\sqcap}(u) \sqcap \hat{X}^{c} \neq \emptyset$, then $\hbar_{j}^{\sqcap}(u) \sqsubseteq \hat{X}$, hence $w \in \hat{X}$. Therefore $\Re_{\aleph_{j}}^{+7}\left(\Re_{\aleph_{j}}^{-7}(\hat{X})\right) \sqsubseteq \hat{X}$.
(U10) From (U7) and (U9).

The equality of (L4), (U3), and (K) in Proposition 4.5 does not hold as shown in the following example.

Example 4.6. Let $\mathcal{U}=\{1,2,3,4\}$ and $\Re \sqsubseteq \mathcal{U} \times \mathcal{U}$ be a reflexive relation on $\mathcal{U}$ defined by

$\Re=\{(1,1),(2,2),(3,3),(4,4),(1,2),(2,1),(3,2)\}$. Take $j=j_{2}$ (and also $j \in\left\{j_{1}, j_{3}, j_{4}, j_{5}, j_{6}, j_{7}, j_{8}\right\}$ are similarly). Then

$$
\hbar_{j_{2}}(1)=\hbar_{j_{2}}(2)=\{1,2\},
$$

$$
\hbar_{j_{2}}(3)=\{3\}, \hbar_{j_{2}}(4)=\{4\} .
$$

Let $\hat{X}_{1}=\{1,3\}$ and $\hat{X}_{2}=\{2\}$. Then $\Re_{\aleph_{j}}^{-7}\left(\hat{X}_{1}\right)=\{3\}, \Re_{\aleph_{j}}^{-7}\left(\hat{X}_{2}\right)=$ $\emptyset, \Re_{\aleph_{j}}^{+7}\left(\hat{X}_{1}\right)=\{1,2,3\}, \Re_{\aleph_{j}}^{+7}\left(\hat{X}_{2}\right)=\{1,2\}, \Re_{\aleph_{j}}^{-7}\left(\hat{X}_{1} \sqcup \hat{X}_{2}\right)=$ $\{1,2,3\}$ and $\Re_{\aleph_{j}}^{+7}\left(\hat{X}_{1} \sqcap \hat{X}_{2}\right)=\emptyset$. Then $\Re_{\aleph_{j}}^{-7}\left(\hat{X}_{1} \sqcup \hat{X}_{2}\right) \neq \Re_{\aleph_{j}}^{-7}\left(\hat{X}_{1}\right)$ $\sqcup \Re_{\aleph_{j}}^{-7}\left(\hat{X}_{2}\right)$ and $\Re_{\aleph_{j}}^{+7}\left(\hat{X}_{1} \sqcap \hat{X}_{2}\right) \neq \Re_{\aleph_{j}}^{+7}\left(\hat{X}_{1}\right) \sqcap \Re_{\aleph_{j}}^{+7}\left(\hat{X}_{2}\right)$. Let $\hat{X}_{1}=\{2,3\}$ and $\hat{X}_{2}=\{3,4\}$, then $\Re_{\aleph_{j}}^{-7}\left(\hat{X}_{1}^{c} \sqcup \hat{X}_{2}\right)=\{3,4\}$ and $\left(\Re_{\aleph_{j}}^{-7}\left(\hat{X}_{1}\right)\right)^{c} \sqcup \Re_{\aleph_{j}}^{-7}\left(\hat{X}_{2}\right)=\mathcal{U}$. Thus $\Re_{\aleph_{j}}^{-7}\left(\hat{X}_{1}^{c} \sqcup \hat{X}_{2}\right) \neq\left(\Re_{\aleph_{j}}^{-7}\left(\hat{X}_{1}\right)\right)^{c} \sqcup$ $\Re_{\aleph_{j}}^{-7}\left(\hat{X}_{2}\right)$.

Definition 4.7. Let $\left(\mathcal{U}, \Re, \vartheta_{j}\right)$ be a $j-\mathbb{R} \mathbb{N} \mathbb{S}$. A reflexive $8 j$ adhesion neighborhood rough set of $\hat{X} \sqsubseteq \mathcal{U}$ in $\left(\mathcal{U}, \Re, \vartheta_{j}\right)$ or with respect to $\vartheta_{j}$ is a pair $\left(\Re_{\aleph_{j}}^{-8}(\hat{X}), \Re_{\aleph_{j}}^{+8}(\hat{X})\right)$, defined by

$$
\Re_{\aleph_{j}}^{-8}(\hat{X})=\bigsqcup\left\{\hbar_{j}^{\Gamma}(u): \hbar_{j}^{\Gamma}(u) \sqsubseteq \hat{X}\right\}
$$

(called the reflexive 8-lower approximation of $\hat{X}$ ) and

$$
\Re_{\aleph_{j}}^{+8}(\hat{X})=\left[\Re_{\aleph_{j}}^{-8}\left(\hat{X}^{c}\right)\right]^{c}
$$

(called the reflexive 8-upper approximation of $\hat{X}$ ).

Example 4.8 (Continued from Example 4.2). Take $j=j_{1}$ (and also $j \in\left\{j_{2}, j_{3}, j_{4}, j_{5}, j_{6}, j_{7}, j_{8}\right\}$ are similarly). If $\hat{X}=$ $\{1,3\}$, then

$$
\Re_{\aleph_{j_{1}}}^{-8}(\hat{X})=\Re_{\aleph_{j_{1}}}^{+8}(\hat{X})=\{1,3\}
$$

Proposition 4.9. Let $\hat{X}, \hat{X}_{1}, \hat{X}_{2} \sqsubseteq \mathcal{U}$. Then the following properties (L1)-(L10), (U1)-(U10), (K), and (LU) will be satisfy based on Definition 4.7.

Proof. Similar to Proposition 4.5.

The equality of (L4), (U3), and (K) in Proposition 4.7 does not hold as shown in the following example.

Example 4.10 (Continued from Example 4.6). Take $j=j_{2}$ (and also $j \in\left\{j_{1}, j_{3}, j_{4}, j_{5}, j_{6}, j_{7}, j_{8}\right\}$ are similarly). Let $\hat{X}_{1}=$ $\{1,3\}$ and $\hat{X}_{2}=\{2\}$. Then $\Re_{\aleph_{j}}^{-8}\left(\hat{X}_{1}\right)=\{3\}, \Re_{\aleph_{j}}^{-8}\left(\hat{X}_{2}\right)=\emptyset$, $\Re_{\aleph_{j}}^{+8}\left(\hat{X}_{1}\right)=\{1,2,3\}, \Re_{\aleph_{j}}^{+8}\left(\hat{X}_{2}\right)=\{1,2\}, \Re_{\aleph_{j}}^{-8}\left(\hat{X}_{1} \sqcup \hat{X}_{2}\right)=$ $\{1,2,3\}$ and $\Re_{\aleph_{j}}^{+8}\left(\hat{X}_{1} \sqcap \hat{X}_{2}\right)=\emptyset$. Then $\Re_{\aleph_{j}}^{-8}\left(\hat{X}_{1} \sqcup \hat{X}_{2}\right) \neq \Re_{\aleph_{j}}^{-8}\left(\hat{X}_{1}\right)$ $\sqcup \Re_{\aleph_{j}}^{-8}\left(\hat{X}_{2}\right)$ and $\Re_{\aleph_{j}}^{+8}\left(\hat{X}_{1} \sqcap \hat{X}_{2}\right) \neq \Re_{\aleph_{j}}^{+8}\left(\hat{X}_{1}\right) \sqcap \Re_{\aleph_{j}}^{+8}\left(\hat{X}_{2}\right)$. Let $\hat{X}_{1}=\{2,3\}$ and $\hat{X}_{2}=\{3,4\}$, then $\Re_{\aleph_{j}}^{-8}\left(\hat{X}_{1}^{c} \sqcup \hat{X}_{2}\right)=\{3,4\}$ and $\left(\Re_{\aleph_{j}}^{-8}\left(\hat{X}_{1}\right)\right)^{c} \sqcup \Re_{\aleph_{j}}^{-8}\left(\hat{X}_{2}\right)=\mathcal{U}$. Thus $\Re_{\aleph_{j}}^{-8}\left(\hat{X}_{1}^{c} \sqcup \hat{X}_{2}\right) \neq\left(\Re_{\aleph_{j}}^{-8}\left(\hat{X}_{1}\right)\right)^{c} \sqcup$ $\Re_{\aleph_{j}}^{-8}\left(\hat{X}_{2}\right)$. 
Definition 4.11. Let $\left(\mathcal{U}, \Re, \vartheta_{j}\right)$ be a $j-\mathbb{R} \mathbb{N} \mathbb{S}$. A reflexive 9 $j$-adhesion neighborhood rough set of $\hat{X} \sqsubseteq \mathcal{U}$ in $\left(\mathcal{U}, \Re, \vartheta_{j}\right)$ or with respect to $\vartheta_{j}$ is a pair $\left(\Re_{\aleph_{j}}^{-9}(\hat{X}), \Re_{\aleph_{j}}^{+9}(\overline{\hat{X}})\right)$, defined by

$$
\Re_{\aleph_{j}}^{-9}(\hat{X})=\left[\Re_{\aleph_{j}}^{+9}\left(\hat{X}^{c}\right)\right]^{c}
$$

(called the reflexive 9-lower approximation of $\hat{X}$ )

and

$$
\Re_{\aleph_{j}}^{+9}(\hat{X})=\bigsqcup\left\{\hbar_{j}^{\sqcap}(u): \hbar_{j}^{\Gamma}(u) \sqcap \hat{X} \neq \emptyset\right\}
$$

called the reflexive 9-upper approximation of $\hat{X}$ ).

Example 4.12 (Continued from Example 4.2). Take $j=j_{1}$ (and also $j \in\left\{j_{2}, j_{3}, j_{4}, j_{5}, j_{6}, j_{7}, j_{8}\right\}$ are similarly). If $\hat{X}=$ $\{1,3\}$, then we have

$$
\Re_{\aleph_{j_{1}}}^{-9}(\hat{X})=\{1,3\}, \quad \Re_{\aleph_{j_{1}}^{+9}}^{+\hat{X}}=\{1,3\} .
$$

Proposition 4.13. Let $\hat{X}, \hat{X}_{1}, \hat{X}_{2} \sqsubseteq \mathcal{U}$. Then the following properties (L1)-(L10), (U1)-(U10), (K), and (LU) will be satisfy based on Definition 4.11.

Proof. Similar to Proposition 4.5.

The equality of (L4), (U3), and (K) in Proposition 4.13 does not hold as shown in the following example.

Example 4.14. (Continued from Example 4.6). Take $j=j_{2}$ (and also $j \in\left\{j_{1}, j_{3}, j_{4}, j_{5}, j_{6}, j_{7}, j_{8}\right\}$ are similarly). Let $\hat{X}_{1}=$ $\{1,3\}$ and $\hat{X}_{2}=\{2\}$. Then $\Re_{\aleph_{j}}^{-9}\left(\hat{X}_{1}\right)=\{3\}, \Re_{\aleph_{j}}^{-9}\left(\hat{X}_{2}\right)=\emptyset$, $\Re_{\aleph_{j}}^{+9}\left(\hat{X}_{1}\right)=\{1,2,3\}, \Re_{\aleph_{j}}^{+9}\left(\hat{X}_{2}\right)=\{1,2\}, \Re_{\aleph_{j}}^{-9}\left(\hat{X}_{1} \sqcup \hat{X}_{2}\right)=$ $\{1,2,3\}$ and $\Re_{\aleph_{j}}^{+9}\left(\hat{X}_{1} \sqcap \hat{X}_{2}\right)=\emptyset$. Then $\Re_{\aleph_{j}}^{-9}\left(\hat{X}_{1} \sqcup \hat{X}_{2}\right) \neq \Re_{\aleph_{j}}^{-9}\left(\hat{X}_{1}\right)$ $\sqcup \Re_{\aleph_{j}}^{-9}\left(\hat{X}_{2}\right)$ and $\Re_{\aleph_{j}}^{+9}\left(\hat{X}_{1} \sqcap \hat{X}_{2}\right) \neq \Re_{\aleph_{j}}^{+9}\left(\hat{X}_{1}\right) \sqcap \Re_{\aleph_{j}}^{+9}\left(\hat{X}_{2}\right)$. Let $\hat{X}_{1}=\{2,3\}$ and $\hat{X}_{2}=\{3,4\}$, then $\Re_{\aleph_{j}}^{-9}\left(\hat{X}_{1}^{c} \sqcup \hat{X}_{2}\right)=\{3,4\}$ and $\left(\Re_{\aleph_{j}}^{-9}\left(\hat{X}_{1}\right)\right)^{c} \sqcup \Re_{\aleph_{j}}^{-9}\left(\hat{X}_{2}\right)=\mathcal{U}$. Thus $\Re_{\aleph_{j}}^{-9}\left(\hat{X}_{1}^{c} \sqcup \hat{X}_{2}\right) \neq\left(\Re_{\aleph_{j}}^{-9}\left(\hat{X}_{1}\right)\right)^{c} \sqcup$ $\Re_{\aleph_{j}}^{-9}\left(\hat{X}_{2}\right)$.

In the following, we will give the comparison between the properties of rough sets based on Definitions 3.3, 3.8, and 3.13 by using j- reflexive neighborhood space and Definitions 4.3, 4.7, and 4.11 by using $\mathrm{j}$-reflexive adhesion neighborhood space as shown in Table 1. 
Table 1 Comparison between the properties of rough sets based on Definitions 3.3, 3.8, and 3.13 by using j-reflexive neighborhood space and Definitions 4.3, 4.7 , and 4.11 by using j-reflexive adhesion neighborhood space.

\begin{tabular}{|c|c|c|c|c|c|c|}
\hline Property & Definition 3.3 & Definition 3.8 & Definition 3.13 & Definition 4.3 & Definition 4.7 & Definition 4.11 \\
\hline L1 & $x$ & $x$ & $x$ & $x$ & $\times$ & $x$ \\
\hline L2 & $x$ & $\times$ & $x$ & $x$ & $\times$ & $x$ \\
\hline L3 & $x$ & $\times$ & $x$ & $x$ & $\times$ & $x$ \\
\hline L4 & $x$ & $x$ & $x$ & $x$ & $x$ & $x$ \\
\hline L5 & $x$ & $\times$ & $x$ & $\times$ & $\times$ & $x$ \\
\hline L6 & $x$ & $x$ & $x$ & $x$ & $x$ & $x$ \\
\hline L7 & $x$ & $x$ & $x$ & $x$ & $\times$ & $x$ \\
\hline L8 & & & $x$ & $\times$ & $x$ & $x$ \\
\hline L9 & $x$ & $x$ & & $\times$ & $x$ & $x$ \\
\hline L10 & & & & $\times$ & $\times$ & $x$ \\
\hline U1 & $x$ & $x$ & $x$ & $x$ & $x$ & $x$ \\
\hline $\mathrm{U} 2$ & $x$ & $x$ & $x$ & $x$ & $x$ & $x$ \\
\hline U3 & $x$ & $x$ & $x$ & $x$ & $x$ & $x$ \\
\hline U4 & $x$ & $\times$ & $x$ & $x$ & $\times$ & $x$ \\
\hline U5 & $x$ & $\times$ & $x$ & $\times$ & $x$ & $x$ \\
\hline U6 & $\times$ & $\times$ & $x$ & $\times$ & $\times$ & $x$ \\
\hline U7 & $x$ & $\times$ & $x$ & $\times$ & $x$ & $x$ \\
\hline U8 & & & $x$ & $x$ & $\times$ & $x$ \\
\hline U9 & $x$ & $\times$ & & $\times$ & $\times$ & $x$ \\
\hline U10 & & & & $\times$ & $x$ & $x$ \\
\hline $\mathrm{K}$ & $x$ & $\times$ & $\times$ & $x$ & $\times$ & $x$ \\
\hline LU & $x$ & $\times$ & $\times$ & $\times$ & $x$ & $x$ \\
\hline
\end{tabular}

\subsection{Fourth kind: Generalized rough set models by based on j-similarity adhesion neighborhood space}

Definition 4.15. Let $\Re$ be similarity on $\mathcal{U}$. The $j$-similarity adhesion neighborhood of element $u \in \mathcal{U}$ are defined as follows: $\forall j \in\left\{j_{1}, j_{2}, j_{3}, j_{4}, j_{5}, j_{6}, j_{7}, j_{8}\right\}$

(1) $\hbar_{j_{1}}(u)=\left\{v \in \mathcal{U}: \aleph \dot{j}_{1}(u)=\aleph \aleph_{j_{1}}(v)\right\}$.

(2) $\hbar_{j_{2}}(u)=\left\{v \in \mathcal{U}: \aleph \aleph_{j_{2}}^{\sqcup}(u)=\aleph \aleph_{j_{2}}^{ப}(v)\right\}$.

(3) $\hbar_{j_{3}}(u)=\left\{v \in \mathcal{U}: \bigcap_{u \in \aleph_{j_{1}}(v)} \aleph_{j_{1}}^{\sqcup}(v)=\bigcap_{v \in \aleph_{j_{1}}(x)} \aleph_{j_{1}}^{\sqcup}(u)\right\}$.

(4) $\hbar_{j_{4}}(u)=\left\{v \in \mathcal{U}: \bigcap_{u \in \aleph_{j_{2}}(v)} \aleph_{j_{2}}^{\sqcup}(v)=\bigcap_{v \in \aleph_{j_{2}}(u)}^{\sqcup} \aleph_{j_{2}}^{\sqcup}(u)\right\}$.

(5) $\hbar_{j_{5}}(u)=\hbar_{j_{1}}(u) \sqcup \hbar_{j_{2}}(u)$.

(6) $\hbar_{j_{6}}(u)=\hbar_{j_{1}}(u) \sqcap \hbar_{j_{2}}(u)$.

(7) $\hbar_{j_{7}}(u)=\hbar_{j_{3}}(u) \sqcup \hbar_{j_{4}}(u)$.

(8) $\hbar_{j_{8}}(u)=\hbar_{j_{3}}(u) \sqcap \hbar_{j_{4}}(u)$.

According to above Definition 4.15 , we call $\left(\mathcal{U}, \Re, \vartheta_{j}\right)$ be a $j$-similarity adhesion neighborhood space (briefly, a $j$-SANS).

Example 4.16 (Continued from Example 4.2). Take $j \in\left\{j_{1}, j_{2}\right.$, $\left.j_{3}, j_{4}, j_{5}, j_{6}, j_{7}, j_{8}\right\}$, Then the $j$-similarity adhesion neighborhood is

$$
\begin{gathered}
\hbar_{j}(1)=\hbar_{j}(2)=\{1,2\}, \\
\hbar_{j}(3)=\{3\}, \quad \hbar_{j}(4)=\{4\} .
\end{gathered}
$$

Definition 4.17. Let $\left(\mathcal{U}, \Re, \vartheta_{j}\right)$ be a $j$-SANS. A similarity 10 $j$-adhesion neighborhood rough set of $\hat{X} \sqsubseteq \mathcal{U}$ in $\left(\mathcal{U}, \Re, \vartheta_{j}\right)$ or with respect to $\vartheta_{j}$ is a pair $\left(\Re_{\aleph_{j}}^{-10}(\hat{X}), \Re_{\aleph_{j}}^{+1 \bar{x}}(\hat{X})\right)$, defined by

$$
\Re_{\aleph_{j}}^{-10}(\hat{X})=\left\{u \in \mathcal{U}: \hbar_{j}^{\sqcup}(u) \sqsubseteq \hat{X}\right\}
$$

(called the similarity 10-lower approximation of $\hat{X}$ ) and

$$
\Re_{\aleph_{j}}^{+10}(\hat{X})=\left\{u \in \mathcal{U}: \hbar_{j}^{\sqcap}(u) \sqcap \hat{X} \neq \emptyset\right\}
$$

(called the similarity 10-upper approximation of $\hat{X}$ ).

Example 4.18 (Continued from Example 4.16). Take $j \in$ $\left\{j_{1}, j_{2}, j_{3}, j_{4}, j_{5}, j_{6}, j_{7}, j_{8}\right\}$. If $\hat{X}=\{1,3\}$, then

$$
\Re_{\aleph_{j}}^{-10}(\hat{X})=\{3\}, \quad \Re_{\aleph_{j}}^{+10}(\hat{X})=\{1,2,3\} .
$$

Proposition 4.19. Let $\hat{X}, \hat{X}_{1}, \hat{X}_{2} \sqsubseteq \mathcal{U}$. Then the following properties (L1)-(L10), (U1)-(U10), (K), and (LU) will be satisfy based on Definition 4.17.

Proof. We only prove (L9), (L10), (U9), and (U10).

(L9) Let $u \in \hat{X}$, for $v \in \hbar_{j}^{\sqcup}(u)$, then we have $u \in \hbar_{j}^{\sqcup}(v)$. Hence, $\hat{X} \cap \hbar_{j}^{\sqcup}(u) \neq \emptyset$. It follows that $v \in \Re_{\aleph_{j}}^{+10}(\hat{X})$. Thus, $\hbar_{j}^{\sqcup}(u) \sqsubseteq \Re_{\aleph_{j}}^{+10}(\hat{X})$ and $u \in \Re_{\aleph_{j}}^{-10}\left(\Re_{\aleph_{j}}^{+10}(\hat{X})\right)$.

(L10) From (L7) and (L9).

(U9) Let $u \in \Re_{\aleph_{j}}^{+10}\left(\Re_{\aleph_{j}}^{-10}(\hat{X})\right)$. Then there exist $v \in \mathcal{U}$ such that $v \in \hbar_{j}^{\sqcup}(u)$ and $\hbar_{j}^{\sqcup}(u) \sqcap \Re_{\aleph_{j}}^{-10}(\hat{X}) \neq \emptyset$. Hence, there exist $w \in$ $\mathcal{U}$ such that $w \in \hbar_{j}^{\sqcup}(u)$ and $w \in \Re_{\aleph_{j}}^{-10}(\hat{X})$, thus $w \notin \Re_{\aleph_{j}}^{+10}\left(\hat{X}^{c}\right)$ and $\hbar_{j}^{\sqcup}(u) \sqcap \hat{X}^{c} \neq \emptyset$, then $\hbar_{j}^{\sqcup}(u) \sqsubseteq \hat{X}$, hence $w \in \hat{X}$. Therefore $\Re_{\aleph_{j}}^{+10}\left(\Re_{\aleph_{j}}^{-10}(\hat{X})\right) \sqsubseteq \hat{X}$

(U10) From (U7) and (U9).

The equality of (L4), (U3), and (K) in Proposition 4.19 does not hold as shown in the following example.

Example 4.20. (Continued from Example 4.16). Take $j=j_{1}$ (and also $j \in\left\{j_{2}, j_{3}, j_{4}, j_{5}, j_{6}, j_{7}, j_{8}\right\}$ are similarly). Let $\hat{X}_{1}=$ $\{1,3\}$ and $\hat{X}_{2}=\{2\}$. Then $\Re_{\aleph_{j}}^{-10}\left(\hat{X}_{1}\right)=\{3\}, \Re_{\aleph_{j}}^{-10}\left(\hat{X}_{2}\right)=\emptyset$, $\Re_{\aleph_{j}}^{+10}\left(\hat{X}_{1}\right)=\{1,2,3\}, \Re_{\aleph_{j}}^{+10}\left(\hat{X}_{2}\right)=\{1,2\}, \Re_{\aleph_{j}}^{-10}\left(\hat{X}_{1} \sqcup \hat{X}_{2}\right)=$ $\{1,2,3\}$ and $\Re_{\aleph_{j}}^{+10}\left(\hat{X}_{1} \sqcap \hat{X}_{2}\right)=\emptyset$. Then $\Re_{\aleph_{j}}^{-10}\left(\hat{X}_{1} \sqcup \hat{X}_{2}\right) \neq$ $\Re_{\aleph_{j}}^{-10}\left(\hat{X}_{1}\right) \sqcup \Re_{\aleph_{j}}^{-10}\left(\hat{X}_{2}\right)$ and $\Re_{\aleph_{j}}^{+10}\left(\hat{X}_{1} \sqcap \hat{X}_{2}\right) \neq \Re_{\aleph_{j}}^{+10}\left(\hat{X}_{1}\right) \sqcap$ $\Re_{\aleph_{j}}^{+10}\left(\hat{X}_{2}\right)$. Let $\hat{X}_{1}=\{2,3\}$ and $\hat{X}_{2}=\{3,4\}$, then $\Re_{\aleph_{j}}^{-10}\left(\hat{X}_{1}^{c} \sqcup\right.$ $\left.\hat{X}_{2}\right)=\{3,4\}$ and $\left(\Re_{\aleph_{j}}^{-10}\left(\hat{X}_{1}\right)\right)^{c} \sqcup \Re_{\aleph_{j}}^{-10}\left(\hat{X}_{2}\right)=\mathcal{U}$. Thus $\Re_{\aleph_{j}}^{-10}\left(\hat{X}_{1}^{c} \sqcup\right.$ $\left.\hat{X}_{2}\right) \neq\left(\Re_{\aleph_{j}}^{-10}\left(\hat{X}_{1}\right)\right)^{c} \sqcup \Re_{\aleph_{j}}^{-10}\left(\hat{X}_{2}\right)$.

Definition 4.21. Let $\left(\mathcal{U}, \Re, \vartheta_{j}\right)$ be a $j$-SANS. A similarity 11 $j$-adhesion neighborhood rough set of $\hat{X} \sqsubseteq \mathcal{U}$ in $\left(\mathcal{U}, \Re, \vartheta_{j}\right)$ or with respect to $\vartheta_{j}$ is a pair $\left(\Re_{\aleph_{j}}^{-11}(\hat{X}), \Re_{\aleph_{j}}^{+11}(\hat{X})\right)$, defined by

$$
\Re_{\aleph_{j}}^{-11}(\hat{X})=\bigsqcup\left\{\hbar_{j}^{\sqcup}(u): \hbar_{j}^{\sqcup}(u) \sqsubseteq \hat{X}\right\}
$$

(called the similarity 11-lower approximation of $\hat{X}$ ) 
and

$$
\Re_{\aleph_{j}}^{+11}(\hat{X})=\left[\Re_{\aleph_{j}}^{-11}\left(\hat{X}^{c}\right)\right]^{c}
$$

called the similarity 11-upper approximation of $\hat{X}$ ).

Example 4.22 (Continued from Example 4.16). Take $j=j_{1}$ (and also $j \in\left\{j_{2}, j_{3}, j_{4}, j_{5}, j_{6}, j_{7}, j_{8}\right\}$ are similarly). If $\hat{X}=$ $\{1,3\}$, then

$$
\Re_{\hbar_{j_{1}}}^{-11}(\hat{X})=\{3\}, \Re_{\hbar_{j_{1}}}^{+11}(\hat{X})=\{1,2,3\} .
$$

Proposition 4.23. Let $\hat{X}, \hat{X}_{1}, \hat{X}_{2} \sqsubseteq \mathcal{U}$. Then the following properties (L1)-(L10), (U1)-(U10), (K), and (LU) will be satisfy based on Definition 4.21.

Proof. Similar to Proposition 4.19.

The equality of (L4), (U3), and (K) in Proposition 4.23 does not hold as shown in the following example.

Example 4.24. (Continued from Example 4.16). Take $j=j_{1}$ (and also $j \in\left\{j_{2}, j_{3}, j_{4}, j_{5}, j_{6}, j_{7}, j_{8}\right\}$ are similarly). Let $\hat{X}_{1}=$ $\{1,3\}$ and $\hat{X}_{2}=\{2\}$. Then $\Re_{\aleph_{j}}^{-11}\left(\hat{X}_{1}\right)=\{3\}, \Re_{\aleph_{j}}^{-11}\left(\hat{X}_{2}\right)=\emptyset$, $\Re_{\aleph_{j}}^{+11}\left(\hat{X}_{1}\right)=\{1,2,3\}, \Re_{\aleph_{j}}^{+11}\left(\hat{X}_{2}\right)=\{1,2\}, \Re_{\aleph_{j}}^{-11}\left(\hat{X}_{1} \sqcup \hat{X}_{2}\right)=$ $\{1,2,3\}$ and $\Re_{\aleph_{j}}^{+11}\left(\hat{X}_{1} \sqcap \hat{X}_{2}\right)=\emptyset$. Then $\Re_{\aleph_{j}}^{-11}\left(\hat{X}_{1} \sqcup \hat{X}_{2}\right) \neq$ $\Re_{\aleph_{j}}^{-11}\left(\hat{X}_{1}\right) \sqcup \Re_{\aleph_{j}}^{-11}\left(\hat{X}_{2}\right)$ and $\Re_{\aleph_{j}}^{+11}\left(\hat{X}_{1} \sqcap \hat{X}_{2}\right) \neq \Re_{\aleph_{j}}^{+11}\left(\hat{X}_{1}\right) \sqcap$ $\Re_{\aleph_{j}}^{+11}\left(\hat{X}_{2}\right)$. Let $\hat{X}_{1}=\{2,3\}$ and $\hat{X}_{2}=\{3,4\}$, then $\Re_{\aleph_{j}}^{-11}\left(\hat{X}_{1}^{c} \sqcup\right.$ $\left.\hat{X}_{2}\right)=\{3,4\}$ and $\left(\Re_{\aleph_{j}}^{-11}\left(\hat{X}_{1}\right)\right)^{c} \sqcup \Re_{\aleph_{j}}^{-11}\left(\hat{X}_{2}\right)=\mathcal{U}$. Thus $\Re_{\aleph_{j}}^{-11}\left(\hat{X}_{1}^{c} \sqcup\right.$ $\left.\hat{X}_{2}\right) \neq\left(\Re_{\aleph_{j}}^{-11}\left(\hat{X}_{1}\right)\right)^{c} \sqcup \Re_{\aleph_{j}}^{-11}\left(\hat{X}_{2}\right)$.

Definition 4.25. Let $\left(\mathcal{U}, \Re, \vartheta_{j}\right)$ be a $j$-SANS. A similarity 12 $j$ - adhesion neighborhood rough set of $\hat{X} \sqsubseteq \mathcal{U}$ in $\left(\mathcal{U}, \Re, \vartheta_{j}\right)$ or with respect to $\vartheta_{j}$ is a pair $\left(\Re_{\aleph_{j}}^{-12}(\hat{X}), \Re_{\aleph_{j}}^{+12}(\hat{X})\right)$, defined by

$$
\Re_{\aleph_{j}}^{-12}(\hat{X})=\left[\Re_{\aleph_{j}}^{+12}\left(\hat{X}^{c}\right)\right]^{c}
$$

(called the similarity 12-lower approximation of $\hat{X}$ )

and

$$
\Re_{\aleph_{j}}^{+12}(\hat{X})=\bigsqcup\left\{\hbar_{j}^{\sqcup}(u): \hbar_{j}^{\sqcup}(u) \sqcap \hat{X} \neq \emptyset\right\}
$$

called the similarity 12-upper approximation of $\hat{X}$ ).

Example 4.26 (Continued from Example 4.16). Take $j=j_{1}$ (and also $j \in\left\{j_{2}, j_{3}, j_{4}, j_{5}, j_{6}, j_{7}, j_{8}\right\}$ are similarly). If $\hat{X}=$ $\{1,3\}$, then we have

$$
\Re_{\aleph_{j_{1}}}^{-12}(\hat{X})=\{3\}, \Re_{\aleph_{j_{1}}}^{+12}(\hat{X})=\{1,2,3\} .
$$

Proposition 4.27. Let $\hat{X}, \hat{X}_{1}, \hat{X}_{2} \sqsubseteq \mathcal{U}$. Then the following properties (L1)-(L10), (U1)-(U10), (K), and (LU) will be satisfy based on Definition 4.25.

Proof. Similar to Proposition 4.19.

The equality of (L4), (U3), and (K) in Proposition 4.27 does not hold as shown in the following example.

Example 4.28. (Continued from Example 4.16). Take $j=j_{1}$ (and also $j \in\left\{j_{2}, j_{3}, j_{4}, j_{5}, j_{6}, j_{7}, j_{8}\right\}$ are similarly). Let $\hat{X}_{1}=$ $\{1,3\}$ and $\hat{X}_{2}=\{2\}$. Then $\Re_{\aleph_{j}}^{-12}\left(\hat{X}_{1}\right)=\{3\}, \Re_{\aleph_{j}}^{-12}\left(\hat{X}_{2}\right)=\emptyset$, $\Re_{\aleph_{j}}^{+12}\left(\hat{X}_{1}\right)=\{1,2,3\}, \Re_{\aleph_{j}}^{+12}\left(\hat{X}_{2}\right)=\{1,2\}, \Re_{\aleph_{j}}^{-12}\left(\hat{X}_{1} \sqcup \hat{X}_{2}\right)=$ $\{1,2,3\}$ and $\Re_{\aleph_{j}}^{+12}\left(\hat{X}_{1} \sqcap \hat{X}_{2}\right)=\emptyset$. Then $\Re_{\aleph_{j}}^{-12}\left(\hat{X}_{1} \sqcup \hat{X}_{2}\right) \neq$ $\Re_{\aleph_{j}}^{-12}\left(\hat{X}_{1}\right) \sqcup \Re_{\aleph_{j}}^{-12}\left(\hat{X}_{2}\right)$ and $\Re_{\aleph_{j}}^{+12}\left(\hat{X}_{1} \sqcap \hat{X}_{2}\right) \neq \Re_{\aleph_{j}}^{+12}\left(\hat{X}_{1}\right) \sqcap$
$\Re_{\aleph_{j}}^{+12}\left(\hat{X}_{2}\right)$. Let $\hat{X}_{1}=\{2,3\}$ and $\hat{X}_{2}=\{3,4\}$, then $\Re_{\aleph_{j}}^{-12}\left(\hat{X}_{1}^{c} \sqcup\right.$ $\left.\hat{X}_{2}\right)=\{3,4\}$ and $\left(\Re_{\aleph_{j}}^{-12}\left(\hat{X}_{1}\right)\right)^{c} \sqcup \Re_{\aleph_{j}}^{-12}\left(\hat{X}_{2}\right)=\mathcal{U}$. Thus $\Re_{\aleph_{j}}^{-12}\left(\hat{X}_{1}^{c} \sqcup\right.$ $\left.\hat{X}_{2}\right) \neq\left(\Re_{\aleph_{j}}^{-12}\left(\hat{X}_{1}\right)\right)^{c} \sqcup \Re_{\aleph_{j}}^{-12}\left(\hat{X}_{2}\right)$.

In the following, we will give the comparison between the properties of rough sets based on Definitions 3.20, 3.25, and 3.30 by using j- similarly neighborhood space and Definitions 4.17, 4.21 , and 4.25 by using j-similarly adhesion neighborhood space as shown in Table 2 . 
Table 2 Comparison between the properties of rough sets based on Definitions 3.20, 3.25, and 3.30 by using j-similarly neighborhood space and Definitions $4.17,4.21$, and 4.25 by using j-similarly adhesion neighborhood space.

\begin{tabular}{|c|c|c|c|c|c|c|}
\hline Property & Definition 3.20 & Definition 3.25 & Definition 3.30 & Definition 4.17 & Definition 4.21 & Definition 4.25 \\
\hline L1 & $\times$ & $x$ & $\times$ & $x$ & $x$ & $x$ \\
\hline L2 & $\times$ & $x$ & $x$ & $x$ & $\times$ & $x$ \\
\hline L3 & $x$ & $x$ & $x$ & $x$ & $x$ & $\times$ \\
\hline L4 & $x$ & & $\times$ & $x$ & $x$ & $x$ \\
\hline L5 & $x$ & $x$ & $x$ & $x$ & $\times$ & $x$ \\
\hline L6 & $\times$ & $x$ & $x$ & $x$ & $x$ & $x$ \\
\hline L7 & $x$ & $x$ & $x$ & $x$ & $x$ & $x$ \\
\hline L8 & $x$ & & $x$ & $x$ & $x$ & $x$ \\
\hline L10 & & & & $x$ & $\times$ & $x$ \\
\hline U1 & $x$ & $x$ & $x$ & $x$ & $x$ & $x$ \\
\hline U2 & $\times$ & $x$ & $x$ & $x$ & $\times$ & $x$ \\
\hline U3 & $x$ & $x$ & $x$ & $x$ & $x$ & $x$ \\
\hline U4 & $x$ & & $x$ & $x$ & $x$ & $x$ \\
\hline U5 & $x$ & $x$ & $x$ & $x$ & $x$ & $x$ \\
\hline U6 & $\times$ & $x$ & $x$ & $\times$ & $\times$ & $x$ \\
\hline U7 & $x$ & $x$ & $x$ & $x$ & $x$ & $x$ \\
\hline U8 & $x$ & & $x$ & $x$ & $x$ & $x$ \\
\hline U10 & & & & $x$ & $x$ & $x$ \\
\hline $\mathrm{K}$ & $x$ & $x$ & $x$ & $x$ & $x$ & $x$ \\
\hline LU & $x$ & $x$ & $x$ & $x$ & $x$ & $x$ \\
\hline
\end{tabular}

A cross $(\times)$ indicates that the property is satisfied.

\subsection{The relationships among our models}

In this subsection, we give the relationships among our models. From the proposed study, we have the following results.

Proposition 4.29. Let $\left(\mathcal{U}, \Re, \vartheta_{j}\right)$ be a $j-\mathbb{R} \mathbb{N} S, j-\mathbb{R N S}$ and $\hat{X} \sqsubseteq \mathcal{U}$. Then we have the following results.

(1) $\Re_{\aleph_{j}}^{-1}(\hat{X}) \sqsubseteq \Re_{\aleph_{j}}^{-7}(\hat{X}), \Re_{\aleph_{j}}^{+7}(\hat{X}) \sqsubseteq \Re_{\aleph_{j}}^{+1}(\hat{X})$.

(2) $\Re_{\aleph_{j}}^{-2}(\hat{X}) \sqsubseteq \Re_{\aleph_{j}}^{-8}(\hat{X}), \Re_{\aleph_{j}}^{+8}(\hat{X}) \sqsubseteq \Re_{\aleph_{j}}^{+2}(\hat{X})$.

(3) $\Re_{\aleph_{j}}^{-3}(\hat{X}) \sqsubseteq \Re_{\aleph_{j}}^{-9}(\hat{X}), \Re_{\aleph_{j}}^{+9}(\hat{X}) \sqsubseteq \Re_{\aleph_{j}}^{+3}(\hat{X})$.

(4) $\Re_{\aleph_{j}}^{-1}(\hat{X}) \sqsubseteq \hat{X} \sqsubseteq \Re_{\aleph_{j}}^{+1}(\hat{X}), \Re_{\aleph_{j}}^{-7}(\hat{X}) \sqsubseteq \hat{X} \sqsubseteq \Re_{\aleph_{j}}^{+7}(\hat{X})$.

(5) $\Re_{\aleph_{j}}^{-2}(\hat{X}) \sqsubseteq \hat{X} \sqsubseteq \Re_{\aleph_{j}}^{+2}(\hat{X}), \Re_{\aleph_{j}}^{-8}(\hat{X}) \sqsubseteq \hat{X} \sqsubseteq \Re_{\aleph_{j}}^{+8}(\hat{X})$.

(6) $\Re_{\aleph_{j}}^{-3}(\hat{X}) \sqsubseteq \hat{X} \sqsubseteq \Re_{\aleph_{j}}^{+3}(\hat{X}), \Re_{\aleph_{j}}^{-9}(\hat{X}) \sqsubseteq \hat{X} \sqsubseteq \Re_{\aleph_{j}}^{+9}(\hat{X})$.

Proof. Follows from Definitions 3.3, 3.8, 3.13, 4.3, 4.7 and 4.11.

Proposition 4.30. Let $\left(\mathcal{U}, \Re, \vartheta_{j}\right)$ be a $j-\mathbb{S A N S}, j-\mathbb{S N S}$ and $\hat{X} \sqsubseteq \mathcal{U}$. Then we have the following results.

(1) $\Re_{\aleph_{j}}^{-4}(\hat{X}) \sqsubseteq \Re_{\aleph_{j}}^{-10}(\hat{X}), \Re_{\aleph_{j}}^{+10}(\hat{X}) \sqsubseteq \Re_{\aleph_{j}}^{+4}(\hat{X})$.

(2) $\Re_{\aleph_{j}}^{-5}(\hat{X}) \sqsubseteq \Re_{\aleph_{j}}^{-11}(\hat{X}), \Re_{\aleph_{j}}^{+11}(\hat{X}) \sqsubseteq \Re_{\aleph_{j}}^{+5}(\hat{X})$.

(3) $\Re_{\aleph_{j}}^{-6}(\hat{X}) \sqsubseteq \Re_{\aleph_{j}}^{-12}(\hat{X}), \Re_{\aleph_{j}}^{+12}(\hat{X}) \sqsubseteq \Re_{\aleph_{j}}^{+6}(\hat{X})$.

(4) $\Re_{\aleph_{j}}^{-4}(\hat{X}) \sqsubseteq \hat{X} \sqsubseteq \Re_{\aleph_{j}}^{+4}(\hat{X}), \Re_{\aleph_{j}}^{-10}(\hat{X}) \sqsubseteq \hat{X} \sqsubseteq \Re_{\aleph_{j}}^{+10}(\hat{X})$.

(5) $\Re_{\aleph_{j}}^{-5}(\hat{X}) \sqsubseteq \hat{X} \sqsubseteq \Re_{\aleph_{j}}^{+5}(\hat{X}), \Re_{\aleph_{j}}^{-11}(\hat{X}) \sqsubseteq \hat{X} \sqsubseteq \Re_{\aleph_{j}}^{+11}(\hat{X})$.

(6) $\Re_{\aleph_{j}}^{-6}(\hat{X}) \sqsubseteq \hat{X} \sqsubseteq \Re_{\aleph_{j}}^{+6}(\hat{X}), \Re_{\aleph_{j}}^{-12}(\hat{X}) \sqsubseteq \hat{X} \sqsubseteq \Re_{\aleph_{j}}^{+12}(\hat{X})$.

Proof. Follows from Definitions 3.20, 3.25, 3.30, 4.17, 4.21 and 4.25 .

The relationships can be illustrated as the following Tables 3 and 4 . 
Table 3 The relationships between our models which are given in Definitions 3.3, 3.8, 3.13, 4.3, 4.7 and 4.11.

$$
\begin{aligned}
& \Re_{\aleph_{j}}^{-1}(\hat{X}) \quad \longrightarrow \quad \Re_{\aleph_{j}}^{-7}(\hat{X}) \quad \longrightarrow \quad \hat{X} \quad \longrightarrow \quad \Re_{\aleph_{j}}^{+7}(\hat{X}) \quad \longrightarrow \quad \Re_{\aleph_{j}}^{+1}(\hat{X})
\end{aligned}
$$

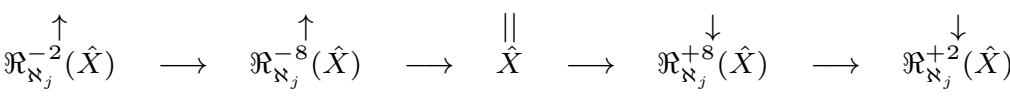

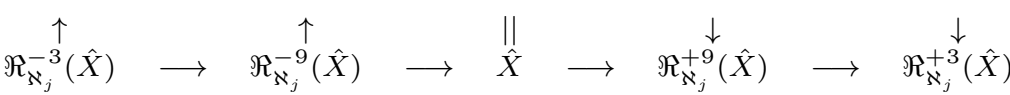

Table 4 The relationships between our models which are given in Definitions 3.20, 3.25, 3.30, 4.17, 4.21 and 4.25.

$$
\begin{aligned}
& \Re_{\aleph_{j}}^{-5}(\hat{X}) \quad \longrightarrow \quad \Re_{\aleph_{j}}^{-11}(\hat{X}) \quad \longrightarrow \quad \hat{X} \quad \longrightarrow \quad \Re_{\aleph_{j}}^{+11}(\hat{X}) \quad \longrightarrow \quad \Re_{\aleph_{j}}^{+5}(\hat{X})
\end{aligned}
$$

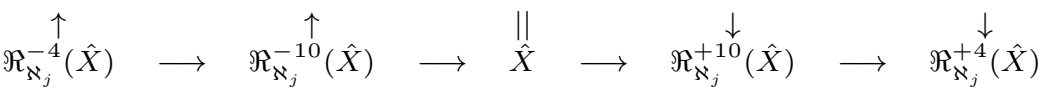

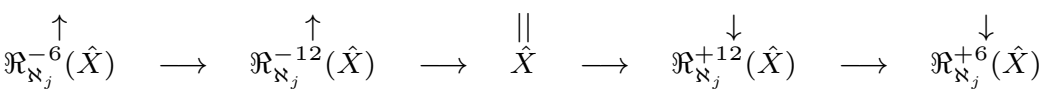

\section{Comparison between the previous methods [24, 25] and the present method}

In this section, we apply the proposed method with a numerical example to describe its performance.

Definition 5.1. Let $\left(\mathcal{U}, \Re, \vartheta_{j}\right)$ be an approximation space and $\hat{X} \sqsubseteq \mathcal{U}$. We define the $i$-boundary region and $i$-accuracy measure, denoted by $\mathcal{B}_{j}^{i}$ and $\mathcal{A}_{j}^{i}$, respectively, defined as follows $(\forall j \in$ $\left\{j_{1}, j_{2}, j_{3}, j_{4}, j_{5}, j_{6}, j_{7}, j_{8}\right\}$ and $\left.\forall i \in\{1,2,3, \ldots, 12\}\right)$ :

(1) $\mathcal{B}_{j}^{i}(\hat{X})=\Re_{\aleph_{j}}^{+i}(\hat{X})-\Re_{\aleph_{j}}^{-i}(\hat{X})$.

(2) $\mathcal{A}_{j}^{i}(\hat{X})=\frac{\left|\Re_{\aleph_{j}}^{-i}(\hat{X})\right|}{\left|\Re_{\aleph_{j}}^{+i}(\hat{X})\right|}$

Based on Definition 5.1, we give the following two examples to illustrate the efficiency for our models.

The main aim of comparison between the previous method and the present method is to increase the accuracy measure and reduce the boundary region of sets by increasing the lower approximations. Moreover, decreasing the upper approximations. Therefore,

(1) We apply Example 5.2 to compare between the Abo Tabl's method [24] (see Definitions 2.4, 2.5, and 2.6) (i.e., Definitions $3.3,3.8$, and 3.13 when $j=j_{1}$ ) and the current method (i.e., Definitions 4.3, 4.7, and 4.11 when $j=j_{1}$ ), respectively.

Example 5.2. Let $\mathcal{U}=\{1,2,3,4\}$ and and $\Re \sqsubseteq \mathcal{U} \times \mathcal{U}$ be a reflexive relation on $\mathcal{U}$ defined by

$$
\begin{aligned}
\Re=\{(1,1),(2,2),(3,3),(4,4),(1,2),(1,3),(2,3),(3,4), \\
(4,2)\} .
\end{aligned}
$$

From Example 5.2, we will compute the reflexive 1 lower approximation, the reflexive 1 upper approximation, the reflexive 1 $j$-neighborhood boundary region, and the reflexive $1 j$-neighborhood accuracy measure for subsets of $\hat{X} \sqsubseteq \mathcal{U}$ in Abo Tabl's method [24] (as in Definition 2.4) (i.e., Definition 3.3 when $j=j_{1}$ ) and the reflexive 7 lower approximation, the reflexive 7 upper approximation, the reflexive $7 j$-adhesion neighborhood boundary region, and the reflexive $7 j$-adhesion neighborhood accuracy measure for subsets of $\hat{X} \sqsubseteq \mathcal{U}$ in the present method in Definition 4.3, respectively, as shown in Table 5. Further, we will compute the reflexive 2 lower approximation, the reflexive 2 upper approxima- tion, the reflexive $2 j$-neighborhood boundary region, and the reflexive $2 j$-neighborhood accuracy measure for subsets of $\hat{X} \sqsubseteq \mathcal{U}$ in Abo Tabl's method [24] (as in Definition 2.5) (i.e., Definition 3.8 when $j=j_{1}$ ) and the reflexive 8 lower approximation, the reflexive 8 upper approximation, the reflexive $8 j$-adhesion neighborhood boundary region, and the reflexive $8 j$-adhesion neighborhood accuracy measure for subsets of $\hat{X} \sqsubseteq \mathcal{U}$ in the present method in Definition 4.7, respectively, as shown in Table 6. Also, we will compute the reflexive 3 lower approximation, the reflexive 3 upper approximation, the reflexive $3 j$-neighborhood boundary region, and the reflexive $3 j$-neighborhood accuracy measure for subsets of $\hat{X} \sqsubseteq \mathcal{U}$ in Abo Tabl's method [24] (as in Definition 2.6) (i.e., Definition 3.13 when $j=j_{1}$ ) and the reflexive 9 lower approximation, the reflexive 9 upper approximation, the reflexive $9 j$-adhesion neighborhood boundary region, and the reflexive $9 j$-adhesion neighborhood accuracy measure for subsets of $\hat{X} \sqsubseteq \mathcal{U}$ in the the present method in Definition 4.11, respectively, as shown in Table 7.

The comparison between the Abo Tabl's method [24] and the present method is shown in Tables 5-7. 
Table 5 Comparison between the reflexive $1 j$-neighborhood boundary region and the reflexive $1 j$-neighborhood accuracy measure of a set $\hat{X}$ by using Abo Tabl's method [24] (as in Definition 2.4) (i.e., Definition 3.3 when $j=j_{1}$ ) and the reflexive $7 j$-adhesion neighborhood boundary region and the reflexive 7 $j$-adhesion neighborhood accuracy measure of a set $\hat{X}$ by using Definition 4.3.

\begin{tabular}{|c|c|c|c|c|c|c|c|c|}
\hline \multirow[t]{2}{*}{$\hat{X}$} & \multicolumn{5}{|c|}{ Abo Tabl's method [24] (as in Definition 2.4) (i.e., Definition 3.3 when $j=j_{1}$ ) } & \multicolumn{3}{|c|}{ The current method in Definition 4.3} \\
\hline & $\Re_{\aleph_{j}}^{-1}(\hat{X})$ & $\Re_{\aleph_{j}}^{+1}(\hat{X})$ & $\mathcal{B}_{j_{1}}^{1}(X)$ & $\mathcal{A}_{j}^{1}(X)$ & $\Re_{\hbar_{j}}^{-7}(\hat{X})$ & $\Re_{\hbar_{j}}^{+7}(\hat{X})$ & $\mathcal{B}_{j_{1}}^{7}(X)$ & $\mathcal{A}_{j}^{7}(X)$ \\
\hline$\emptyset$ & $\emptyset$ & $\emptyset$ & $\emptyset$ & 0 & $\emptyset$ & $\emptyset$ & $\emptyset$ & 0 \\
\hline$\{1\}$ & $\emptyset$ & $\mathcal{U}$ & $\mathcal{U}$ & 0 & $\{1\}$ & $\{1\}$ & $\emptyset$ & 1 \\
\hline$\{2\}$ & $\{2\}$ & $\{1,2\}$ & $\{1\}$ & $\frac{1}{2}$ & $\{2\}$ & $\{2\}$ & $\emptyset$ & 1 \\
\hline$\{3\}$ & $\{3\}$ & $\{1,3\}$ & $\{1\}$ & $\frac{1}{2}$ & $\{3\}$ & $\{3\}$ & $\emptyset$ & 1 \\
\hline$\{4\}$ & $\{4\}$ & $\{4\}$ & $\emptyset$ & 1 & $\{4\}$ & $\{4\}$ & $\emptyset$ & 1 \\
\hline$\{1,2\}$ & $\{2\}$ & $\{1,2\}$ & $\{1\}$ & $\frac{1}{2}$ & $\{1,2\}$ & $\{1,2\}$ & $\emptyset$ & 1 \\
\hline$\{1,3\}$ & $\{3\}$ & $\{1,3\}$ & $\{1\}$ & $\frac{1}{2}$ & $\{1,3\}$ & $\{1,3\}$ & $\emptyset$ & 1 \\
\hline$\{1,4\}$ & $\{4\}$ & $\{1,4\}$ & $\{1\}$ & $\frac{1}{2}$ & $\{1,4\}$ & $\{1,4\}$ & $\emptyset$ & 1 \\
\hline$\{2,3\}$ & $\{2,3\}$ & $\{1,2,3\}$ & $\{1\}$ & $\frac{2}{3}$ & $\{2,3\}$ & $\{2,3\}$ & $\emptyset$ & 1 \\
\hline$\{2,4\}$ & $\{2,4\}$ & $\{1,2,4\}$ & $\{1\}$ & $\frac{2}{3}$ & $\{2,4\}$ & $\{2,4\}$ & $\emptyset$ & 1 \\
\hline$\{3,4\}$ & $\{3,4\}$ & $\{1,3,4\}$ & $\{1\}$ & $\frac{2}{3}$ & $\{3,4\}$ & $\{3,4\}$ & $\emptyset$ & 1 \\
\hline$\{1,2,3\}$ & $\{1,2,3\}$ & $\{1,2,3\}$ & $\emptyset$ & 1 & $\{1,2,3\}$ & $\{1,2,3\}$ & $\emptyset$ & 1 \\
\hline$\{1,2,4\}$ & $\{2,4\}$ & $\{1,2,4\}$ & $\{1\}$ & $\frac{2}{3}$ & $\{1,2,4\}$ & $\{1,2,4\}$ & $\emptyset$ & 1 \\
\hline$\{1,3,4\}$ & $\{3,4\}$ & $\{1,3,4\}$ & $\{1\}$ & $\frac{2}{3}$ & $\{1,3,4\}$ & $\{1,3,4\}$ & $\emptyset$ & 1 \\
\hline$\{2,3,4\}$ & $\{2,3,4\}$ & $\mathcal{U}$ & $\{1\}$ & $\frac{3}{4}$ & $\{2,3,4\}$ & $\{2,3,4\}$ & $\emptyset$ & 1 \\
\hline $\mathcal{U}$ & $\mathcal{U}$ & $\mathcal{U}$ & $\emptyset$ & 1 & $\mathcal{U}$ & $\mathcal{U}$ & $\emptyset$ & 1 \\
\hline
\end{tabular}

Table 6 Comparison between the reflexive $2 j$-neighborhood boundary region and the reflexive $2 j$-neighborhood accuracy measure of a set $\hat{X}$ by using Abo Tabl's method [24] (as in Definition 2.5) (i.e., Definition 3.8 when $j=j_{1}$ ) and the reflexive $8 j$-adhesion neighborhood boundary region and the reflexive 8 $j$-adhesion neighborhood accuracy measure of a set $\hat{X}$ by using Definition 4.7.

\begin{tabular}{|c|c|c|c|c|c|c|c|c|}
\hline \multirow[t]{2}{*}{$\hat{X}$} & \multicolumn{5}{|c|}{ Abo Tabl's method [24] (as in Definition 2.5) (i.e., Definition 3.8 when $j=j_{1}$ ) } & \multicolumn{3}{|c|}{ The current method in Definition 4.7} \\
\hline & $\Re_{\aleph_{j}}^{-2}(\hat{X})$ & $\Re_{\aleph_{j}}^{+2}(\hat{X})$ & $\mathcal{B}_{j_{1}}^{2}(X)$ & $\mathcal{A}_{j}^{2}(X)$ & $\Re_{\hbar_{j}}^{-8}(\hat{X})$ & $\Re_{\hbar_{j}}^{+8}(\hat{X})$ & $\mathcal{B}_{j_{1}}^{8}(X)$ & $\mathcal{A}_{j}^{8}(X)$ \\
\hline$\emptyset$ & $\emptyset$ & $\emptyset$ & $\emptyset$ & 0 & $\emptyset$ & $\emptyset$ & $\emptyset$ & 0 \\
\hline$\{1\}$ & $\emptyset$ & $\{1\}$ & $\{1\}$ & 0 & $\{1\}$ & $\{1\}$ & $\emptyset$ & 1 \\
\hline$\{2\}$ & $\{2\}$ & $\{1,2\}$ & $\{1\}$ & $\frac{1}{2}$ & $\{2\}$ & $\{2\}$ & $\emptyset$ & 1 \\
\hline$\{3\}$ & $\{3\}$ & $\{1,3\}$ & $\{1\}$ & $\frac{2}{2}$ & $\{3\}$ & $\{3\}$ & $\emptyset$ & 1 \\
\hline$\{4\}$ & $\{4\}$ & $\{4\}$ & $\emptyset$ & 1 & $\{4\}$ & $\{4\}$ & $\emptyset$ & 1 \\
\hline$\{1,2\}$ & $\{2\}$ & $\{1,2\}$ & $\{1\}$ & $\frac{1}{2}$ & $\{1,2\}$ & $\{1,2\}$ & $\emptyset$ & 1 \\
\hline$\{1,3\}$ & $\{3\}$ & $\{1,3\}$ & $\{1\}$ & $\frac{1}{2}$ & $\{1,3\}$ & $\{1,3\}$ & $\emptyset$ & 1 \\
\hline$\{1,4\}$ & $\{4\}$ & $\{1,4\}$ & $\{1\}$ & $\frac{1}{2}$ & $\{1,4\}$ & $\{1,4\}$ & $\emptyset$ & 1 \\
\hline$\{2,3\}$ & $\{2,3\}$ & $\{1,2,3\}$ & $\{1\}$ & $\frac{2}{3}$ & $\{2,3\}$ & $\{2,3\}$ & $\emptyset$ & 1 \\
\hline$\{2,4\}$ & $\{2,4\}$ & $\{1,2,4\}$ & $\{1\}$ & $\frac{2}{3}$ & $\{2,4\}$ & $\{2,4\}$ & $\emptyset$ & 1 \\
\hline$\{3,4\}$ & $\{3,4\}$ & $\{1,3,4\}$ & $\{1\}$ & $\frac{2}{3}$ & $\{3,4\}$ & $\{3,4\}$ & $\emptyset$ & 1 \\
\hline$\{1,2,3\}$ & $\{1,2,3\}$ & $\{1,2,3\}$ & $\emptyset$ & 0 & $\{1,2,3\}$ & $\{1,2,3\}$ & $\emptyset$ & 1 \\
\hline$\{1,2,4\}$ & $\{2,4\}$ & $\{1,2,4\}$ & $\{1\}$ & $\frac{2}{3}$ & $\{1,2,4\}$ & $\{1,2,4\}$ & $\emptyset$ & 1 \\
\hline$\{1,3,4\}$ & $\{3,4\}$ & $\{1,3,4\}$ & $\{1\}$ & $\frac{3}{3}$ & $\{1,3,4\}$ & $\{1,3,4\}$ & $\emptyset$ & 1 \\
\hline$\{2,3,4\}$ & $\{2,3,4\}$ & $\mathcal{U}$ & $\{1\}$ & $\frac{3}{4}$ & $\{2,3,4\}$ & $\{2,3,4\}$ & $\emptyset$ & 1 \\
\hline $\mathcal{U}$ & $\mathcal{U}$ & $\mathcal{U}$ & $\emptyset$ & 1 & $\mathcal{U}$ & $\mathcal{U}$ & $\emptyset$ & 1 \\
\hline
\end{tabular}

Table 7 Comparison between the reflexive $3 j$-neighborhood boundary region and the reflexive $3 j$-neighborhood accuracy measure of a set $\hat{X}$ by using Abo Tabl's method [24] (as in Definition 2.6) (i.e., Definition 3.13 when $j=j_{1}$ ) and the reflexive $9 j$-adhesion neighborhood boundary region and the reflexive 9 $j$-adhesion neighborhood accuracy measure of a set $\hat{X}$ by using Definition 4.11 .

\begin{tabular}{|c|c|c|c|c|c|c|c|c|}
\hline \multirow[t]{2}{*}{$\hat{X}$} & \multicolumn{5}{|c|}{ Abo Tabl's method [24] (as in Definition 2.6) (i.e., Definition 3.13 when $j=j_{1}$ ) } & \multicolumn{3}{|c|}{ The current method in Definition 4.11} \\
\hline & $\Re_{\aleph_{j}}^{-3}(\hat{X})$ & $\Re_{\aleph_{j}}^{+3}(\hat{X})$ & $\mathcal{B}_{j_{1}}^{3}(X)$ & $\mathcal{A}_{j}^{3}(X)$ & $\Re_{\hbar_{j}}^{-9}(\hat{X})$ & $\Re_{\hbar_{j}}^{+9}(\hat{X})$ & $\mathcal{B}_{j_{1}}^{9}(X)$ & $\mathcal{A}_{j}^{9}(X)$ \\
\hline$\emptyset$ & $\emptyset$ & $\emptyset$ & $\emptyset$ & 0 & $\emptyset$ & $\emptyset$ & $\emptyset$ & 0 \\
\hline$\{1\}$ & $\emptyset$ & $\{1,2,3\}$ & $\{1,2,3\}$ & 0 & $\{1\}$ & $\{1\}$ & $\emptyset$ & 1 \\
\hline$\{2\}$ & $\emptyset$ & $\{1,2,3\}$ & $\{1,2,3\}$ & 0 & $\{2\}$ & $\{2\}$ & $\emptyset$ & 1 \\
\hline$\{3\}$ & $\emptyset$ & $\{1,2,3\}$ & $\{1,2,3\}$ & 0 & $\{3\}$ & $\{3\}$ & $\emptyset$ & 1 \\
\hline$\{4\}$ & $\{4\}$ & $\{4\}$ & $\emptyset$ & 1 & $\{4\}$ & $\{4\}$ & $\emptyset$ & 1 \\
\hline$\{1,2\}$ & $\emptyset$ & $\{1,2,3\}$ & $\{1,2,3\}$ & 0 & $\{1,2\}$ & $\{1,2\}$ & $\emptyset$ & 1 \\
\hline$\{1,3\}$ & $\emptyset$ & $\{1,2,3\}$ & $\{1,2,3\}$ & 0 & $\{1,3\}$ & $\{1,3\}$ & $\emptyset$ & 1 \\
\hline$\{1,4\}$ & $\{4\}$ & $\mathcal{U}$ & $\{1,2,3\}$ & $\frac{1}{4}$ & $\{1,4\}$ & $\{1,4\}$ & $\emptyset$ & 1 \\
\hline$\{2,3\}$ & $\emptyset$ & $\{1,2,3\}$ & $\{1,2,3\}$ & $\begin{array}{l}4 \\
0\end{array}$ & $\{2,3\}$ & $\{2,3\}$ & $\emptyset$ & 1 \\
\hline$\{2,4\}$ & $\{4\}$ & $\mathcal{U}$ & $\{1,2,3\}$ & $\frac{1}{4}$ & $\{2,4\}$ & $\{2,4\}$ & $\emptyset$ & 1 \\
\hline$\{3,4\}$ & $\{4\}$ & $\mathcal{U}$ & $\{1,2,3\}$ & $\frac{4}{4}$ & $\{3,4\}$ & $\{3,4\}$ & $\emptyset$ & 1 \\
\hline$\{1,2,3\}$ & $\{1,2,3\}$ & $\{1,2,3\}$ & $\emptyset$ & $\begin{array}{l}4 \\
1\end{array}$ & $\{1,2,3\}$ & $\{1,2,3\}$ & $\emptyset$ & 1 \\
\hline$\{1,2,4\}$ & $\{4\}$ & $\mathcal{U}$ & $\{1,2,3\}$ & $\frac{1}{4}$ & $\{1,2,4\}$ & $\{1,2,4\}$ & $\emptyset$ & 1 \\
\hline$\{1,3,4\}$ & $\{4\}$ & $\mathcal{U}$ & $\{1,2,3\}$ & $\begin{array}{l}\frac{4}{4} \\
\frac{1}{4}\end{array}$ & $\{1,3,4\}$ & $\{1,3,4\}$ & $\emptyset$ & 1 \\
\hline$\{2,3,4\}$ & $\{4\}$ & $\mathcal{U}$ & $\{1,2,3\}$ & $\frac{1}{4}$ & $\{2,3,4\}$ & $\{2,3,4\}$ & $\emptyset$ & 1 \\
\hline $\mathcal{U}$ & $\mathcal{U}$ & $\mathcal{U}$ & $\emptyset$ & 1 & $\mathcal{U}$ & $\mathcal{U}$ & $\emptyset$ & 1 \\
\hline
\end{tabular}


From Table 5, for example, if we take $\{1,2\}$, then the reflexive 7 $j$-adhesion neighborhood boundary and the reflexive $7 j$-adhesion neighborhood accuracy by Definition 4.3 are $\emptyset$ and 1 , respectively, whereas the reflexive $1 j$-neighborhood boundary and the reflexive $1 j$-neighborhood accuracy by using Abo Tabl's method [24] (as in Definition 2.4) (i.e., Definition 3.3 when $j=j_{1}$ ) are $\{1\}$ and $\frac{1}{2}$, respectively. Also, from Table 6 , for example, if we take $\{2,3\}$, then the reflexive $8 j$-adhesion neighborhood boundary and the reflexive $8 j$-adhesion neighborhood accuracy by Definition 4.7 are $\emptyset$ and 1 , respectively, whereas the reflexive $2 j$ neighborhood boundary and the reflexive $2 j$-neighborhood accuracy by using Abo Tabl's method [24] (as in Definition 2.5) (i.e., Definition 3.8 when $j=j_{1}$ ) are $\{1\}$ and $\frac{2}{3}$, respectively. Additionally, from Table 7 , for example, if we take $\{1,4\}$, then the reflexive $9 j$-adhesion neighborhood boundary and the reflexive $9 j$-adhesion neighborhood accuracy by Definition 4.11 are $\emptyset$ and 1 , respectively, whereas the reflexive $3 j$-neighborhood boundary and the reflexive $3 j$-neighborhood accuracy by using Abo Tabl's method [24] (as in Definition 2.6) (i.e., Definition 3.13 when $\left.j=j_{1}\right)$ are $\{1,2,3\}$ and $\frac{1}{4}$, respectively.

From Tables 5-7, we can see the reflexive 7-9 of lower and upper approximations based on $j$-adhesion neighborhood space are achieved to increase the accuracy measure and reduce the boundary region of set $\hat{X}$. Therefore, the results by Definitions 4.3, 4.7, and 4.11 when $j=j_{1}$ are better than the Abo Tabl's method [24] (as Definitions 2.4, 2.5, and 2.6) (i.e., Definitions 3.3, 3.8, and 3.13 when $j=j_{1}$ ), respectively.

(2) We apply Example 5.3 to compare between the Dai et al.'s method [25] (see Definitions 2.7, 2.8, and 2.9) (i.e., Definitions $3.20,3.25$, and 3.30 when $j=j_{1}$ ) and the current method (i.e., Definitions 4.17, 4.21, and 4.25 when $j=j_{1}$ ), respectively.

Example 5.3. Let $\mathcal{U}=\{1,2,3,4\}$ and $\Re \sqsubseteq \mathcal{U} \times \mathcal{U}$ be a similarity relation on $\mathcal{U}$ defined by

$$
\begin{aligned}
\Re=\{(1,1),(1,2),(1,4),(2,1),(2,2),(2,3),(3,3),(3,2), & \\
& (4,1),(4,4)\} .
\end{aligned}
$$

From Example 5.3, we will compute the similarity 4 lower approximation, the similarity 4 upper approximation, the similarity $4 j$-neighborhood boundary region, and the similarity $4 j$ neighborhood accuracy measure for subsets of $\hat{X} \sqsubseteq \mathcal{U}$ in Dai et al.'s method [25] (as in Definition 2.8) (i.e., Definition 3.20 when $j=j_{1}$ ) and the similarity 10 lower approximation, the similarity 10 upper approximation, the similarity $10 j$-adhesion neighborhood boundary region, and the similarity $10 j$-adhesion neighborhood accuracy measure for subsets of $\hat{X} \sqsubseteq \mathcal{U}$ in present method in Definition 4.17, respectively, as shown in Table 8. Further, we will compute the similarity 5 lower approximation, the similarity 5 upper approximation, the similarity $5 j$-neighborhood boundary region, and the similarity $5 j$-neighborhood accuracy measure for subsets of $\hat{X} \sqsubseteq \mathcal{U}$ in Dai et al.'s method [25] (as in Definition 2.9) (i.e., Definition 3.25 when $j=j_{1}$ ) and the similarity 11 lower approximation, the similarity 11 upper approximation, the similarity $11 j$-adhesion neighborhood boundary region, and the similarity $11 j$-adhesion neighborhood accuracy measure for subsets of $\hat{X} \sqsubseteq \mathcal{U}$ in the present method in Definition 4.21, respectively, as shown in Table 9. Finally, we will compute the similarity 6 lower approximation, the similarity 6 upper approximation, the similarity $6 j$-neighborhood boundary region, and the similarity 6 $j$-neighborhood accuracy measure for subsets of $\hat{X} \sqsubseteq \mathcal{U}$ in Dai et al.'s method [25] (as in Definition 2.10) (i.e., Definition 3.30 when $j=j_{1}$ ) and the similarity 12 lower approximation, the similarity 12 upper approximation, the similarity $12 j$-adhesion neighborhood boundary region, and the similarity $12 j$-adhesion neighborhood accuracy measure for subsets of $\hat{X} \sqsubseteq \mathcal{U}$ in the present method in Definition 4.25, respectively, as shown in Table 10.

The comparison between the Dai et al.'s method [25] and the present method is shown in Tables 8-10. 
Table 8 Comparison between the similarity $4 j$-neighborhood boundary region and the similarity $4 j$-neighborhood accuracy measure of a set $\hat{X}$ by using Dai et al.'s method [25] (as in Definition 2.8) (i.e., Definition 3.20 when $j=j_{1}$ ) and the similarity $10 j$-adhesion neighborhood boundary region and the similarity $10 j$-adhesion neighborhood accuracy measure of a set $\hat{X}$ by using Definition 4.17 .

\begin{tabular}{|c|c|c|c|c|c|c|c|c|}
\hline \multirow[t]{2}{*}{$\hat{X}$} & \multicolumn{5}{|c|}{ Dai et al.'s method [25] (as in Definition 2.8) (i.e., Definition 3.20 when $j=j_{1}$ ) } & \multicolumn{3}{|c|}{ The current method in Definition 4.17} \\
\hline & $\Re_{\aleph_{j}}^{-4}(\hat{X})$ & $\Re_{\aleph_{j}}^{+4}(\hat{X})$ & $\mathcal{B}_{j_{1}}^{4}(X)$ & $\mathcal{A}_{j}^{4}(X)$ & $\Re_{\hbar_{j}}^{-10}(\hat{X})$ & $\Re_{\hbar_{j}}^{+10}(\hat{X})$ & $\mathcal{B}_{j_{1}}^{10}(X)$ & $\mathcal{A}_{j}^{10}(X)$ \\
\hline$\emptyset$ & $\emptyset$ & $\emptyset$ & $\emptyset$ & 0 & $\emptyset$ & $\emptyset$ & $\emptyset$ & 0 \\
\hline$\{1\}$ & $\emptyset$ & $\mathcal{U}$ & $\mathcal{U}$ & 0 & $\emptyset$ & $\{1,2\}$ & $\{1,2\}$ & 0 \\
\hline$\{2\}$ & $\emptyset$ & $\mathcal{U}$ & $\mathcal{U}$ & 0 & $\emptyset$ & $\{1,2\}$ & $\{1,2\}$ & 0 \\
\hline$\{3\}$ & $\emptyset$ & $\{1,2,3\}$ & $\{1,2,3\}$ & 0 & $\{3\}$ & $\{3\}$ & $\emptyset$ & 1 \\
\hline$\{4\}$ & $\emptyset$ & $\{1,2,4\}$ & $\{1,2,4\}$ & 0 & $\{4\}$ & $\{4\}$ & $\emptyset$ & 1 \\
\hline$\{1,2\}$ & $\emptyset$ & $\mathcal{U}$ & $\mathcal{U}$ & 0 & $\{1,2\}$ & $\{1,2\}$ & $\emptyset$ & 1 \\
\hline$\{1,3\}$ & $\emptyset$ & $\mathcal{U}$ & $\mathcal{U}$ & 0 & $\{3\}$ & $\{1,2,3\}$ & $\{1,2\}$ & $\frac{1}{3}$ \\
\hline$\{1,4\}$ & $\emptyset$ & $\mathcal{U}$ & $\mathcal{U}$ & 0 & $\{4\}$ & $\{1,2,4\}$ & $\{1,2\}$ & $\frac{1}{3}$ \\
\hline$\{2,3\}$ & $\emptyset$ & $\mathcal{U}$ & $\mathcal{U}$ & 0 & $\{3\}$ & $\{1,2,3\}$ & $\{1,2\}$ & $\frac{1}{3}$ \\
\hline$\{2,4\}$ & $\emptyset$ & $\mathcal{U}$ & $\mathcal{U}$ & 0 & $\{4\}$ & $\{1,2,4\}$ & $\{1,2\}$ & $\frac{1}{3}$ \\
\hline$\{3,4\}$ & $\emptyset$ & $\mathcal{U}$ & $\mathcal{U}$ & 0 & $\{3,4\}$ & $\{3,4\}$ & $\emptyset$ & 1 \\
\hline$\{1,2,3\}$ & $\{3\}$ & $\mathcal{U}$ & $\{1,2,4\}$ & $\frac{1}{4}$ & $\{1,2,3\}$ & $\{1,2,3\}$ & $\emptyset$ & 1 \\
\hline$\{1,2,4\}$ & $\{4\}$ & $\mathcal{U}$ & $\{1,2,3\}$ & $\frac{1}{4}$ & $\{1,2,4\}$ & $\{1,2,4\}$ & $\emptyset$ & 1 \\
\hline$\{1,3,4\}$ & $\emptyset$ & $\mathcal{U}$ & $\mathcal{U}$ & 0 & $\{3,4\}$ & $\mathcal{U}$ & $\{1,2\}$ & $\frac{2}{4}$ \\
\hline$\{2,3,4\}$ & $\emptyset$ & $\mathcal{U}$ & $\mathcal{U}$ & 0 & $\{3,4\}$ & $\mathcal{U}$ & $\{1,2\}$ & $\frac{2}{4}$ \\
\hline $\mathcal{U}$ & $\mathcal{U}$ & $\mathcal{U}$ & $\emptyset$ & 1 & $\mathcal{U}$ & $\mathcal{U}$ & $\emptyset$ & 1 \\
\hline
\end{tabular}

Table 9 Comparison between the similarity $5 j$-neighborhood boundary region and the similarity $5 j$-neighborhood accuracy measure of a set $\hat{X}$ by using Dai et al.'s method [25] (as in Definition 2.9) (i.e., Definition 3.25 when $j=j_{1}$ ) and the similarity $11 j$-adhesion neighborhood boundary region and the similarity $11 j$-adhesion neighborhood accuracy measure of a set $\hat{X}$ by using Definition 4.21 .

\begin{tabular}{|c|c|c|c|c|c|c|c|c|}
\hline \multirow[t]{2}{*}{$\hat{X}$} & \multicolumn{5}{|c|}{ Dai et al.'s method [25] (as in Definition 2.9) (i.e., Definition 3.25 when $j=j_{1}$ ) } & \multicolumn{3}{|c|}{ The current method in Definition 4.21} \\
\hline & $\Re_{\aleph_{j}}^{-5}(\hat{X})$ & $\Re_{\aleph_{j}}^{+5}(\hat{X})$ & $\mathcal{B}_{j_{1}}^{5}(X)$ & $\mathcal{A}_{j}^{5}(X)$ & $\Re_{\hbar_{j}}^{-10}(\hat{X})$ & $\Re_{\hbar_{j}}^{+10}(\hat{X})$ & $\mathcal{B}_{j_{1}}^{10}(X)$ & $\mathcal{A}_{j}^{10}(X)$ \\
\hline$\emptyset$ & $\emptyset$ & $\emptyset$ & $\emptyset$ & 0 & $\emptyset$ & $\emptyset$ & $\emptyset$ & 0 \\
\hline$\{1\}$ & $\emptyset$ & $\mathcal{U}$ & $\mathcal{U}$ & 0 & $\emptyset$ & $\{1,2\}$ & $\{1,2\}$ & 0 \\
\hline$\{2\}$ & $\emptyset$ & $\mathcal{U}$ & $\mathcal{U}$ & 0 & $\emptyset$ & $\{1,2\}$ & $\{1,2\}$ & 0 \\
\hline$\{3\}$ & $\emptyset$ & $\{1,2,3\}$ & $\{1,2,3\}$ & 0 & $\{3\}$ & $\{3\}$ & $\emptyset$ & 1 \\
\hline$\{4\}$ & $\emptyset$ & $\{1,2,4\}$ & $\{1,2,4\}$ & 0 & $\{4\}$ & $\{4\}$ & $\emptyset$ & 1 \\
\hline$\{1,2\}$ & $\emptyset$ & $\mathcal{U}$ & $\mathcal{U}$ & 0 & $\{1,2\}$ & $\{1,2\}$ & $\emptyset$ & 1 \\
\hline$\{1,3\}$ & $\emptyset$ & $\mathcal{U}$ & $\mathcal{U}$ & 0 & $\{3\}$ & $\{1,2,3\}$ & $\{1,2\}$ & $\frac{1}{3}$ \\
\hline$\{1,4\}$ & $\emptyset$ & $\mathcal{U}$ & $\mathcal{U}$ & 0 & $\{4\}$ & $\{1,2,4\}$ & $\{1,2\}$ & $\frac{1}{3}$ \\
\hline$\{2,3\}$ & $\emptyset$ & $\mathcal{U}$ & $\mathcal{U}$ & 0 & $\{3\}$ & $\{1,2,3\}$ & $\{1,2\}$ & $\frac{9}{3}$ \\
\hline$\{2,4\}$ & $\emptyset$ & $\mathcal{U}$ & $\mathcal{U}$ & 0 & $\{4\}$ & $\{1,2,4\}$ & $\{1,2\}$ & $\frac{1}{3}$ \\
\hline$\{3,4\}$ & $\emptyset$ & $\mathcal{U}$ & $\mathcal{U}$ & 0 & $\{3,4\}$ & $\{3,4\}$ & $\emptyset$ & 1 \\
\hline$\{1,2,3\}$ & $\{1,2,3\}$ & $\mathcal{U}$ & $\{4\}$ & $\frac{3}{4}$ & $\{1,2,3\}$ & $\{1,2,3\}$ & $\emptyset$ & 1 \\
\hline$\{1,2,4\}$ & $\{1,2,4\}$ & $\mathcal{U}$ & $\{3\}$ & $\frac{3}{4}$ & $\{1,2,4\}$ & $\{1,2,4\}$ & $\emptyset$ & 1 \\
\hline$\{1,3,4\}$ & $\emptyset$ & $\mathcal{U}$ & $\mathcal{U}$ & 0 & $\{3,4\}$ & $\mathcal{U}$ & $\{1,2\}$ & $\frac{2}{4}$ \\
\hline$\{2,3,4\}$ & $\emptyset$ & $\mathcal{U}$ & $\mathcal{U}$ & 0 & $\{3,4\}$ & $\mathcal{U}$ & $\{1,2\}$ & $\frac{2}{4}$ \\
\hline $\mathcal{U}$ & $\mathcal{U}$ & $\mathcal{U}$ & $\emptyset$ & 1 & $\mathcal{U}$ & $\mathcal{U}$ & $\emptyset$ & 1 \\
\hline
\end{tabular}

Table 10 Comparison between the similarity $6 j$-neighborhood boundary region and the similarity $6 j$-neighborhood accuracy measure of a set $\hat{X}$ by using Dai et al.'s method [25] (as in Definition 2.10) (i.e., Definition 3.30 when $j=j_{1}$ ) and the similarity $12 j$-adhesion neighborhood boundary region and the similarity $12 j$-adhesion neighborhood accuracy measure of a set $\hat{X}$ by using Definition 4.25 .

\begin{tabular}{|c|c|c|c|c|c|c|c|c|}
\hline \multirow[t]{2}{*}{$\hat{X}$} & \multicolumn{5}{|c|}{ Dai et al.'s method [25] (as in Definition 2.10) (i.e., Definition 3.30 when $j=j_{1}$ ) } & \multicolumn{3}{|c|}{ The current method in Definition 4.25} \\
\hline & $\Re_{\aleph_{j}}^{-6}(\hat{X})$ & $\Re_{\aleph_{j}}^{+6}(\hat{X})$ & $\mathcal{B}_{j_{1}}^{6}(X)$ & $\mathcal{A}_{j}^{6}(X)$ & $\Re_{\hbar_{j}}^{-12}(\hat{X})$ & $\Re_{\hbar_{j}}^{+12}(\hat{X})$ & $\mathcal{B}_{j_{1}}^{12}(X)$ & $\mathcal{A}_{j}^{12}(X)$ \\
\hline$\emptyset$ & $\emptyset$ & $\emptyset$ & $\emptyset$ & 0 & $\emptyset$ & $\emptyset$ & $\emptyset$ & 0 \\
\hline$\{1\}$ & $\emptyset$ & $\mathcal{U}$ & $\mathcal{U}$ & 0 & $\emptyset$ & $\{1,2\}$ & $\{1,2\}$ & 0 \\
\hline$\{2\}$ & $\emptyset$ & $\mathcal{U}$ & $\mathcal{U}$ & 0 & $\emptyset$ & $\{1,2\}$ & $\{1,2\}$ & 0 \\
\hline$\{3\}$ & $\emptyset$ & $\mathcal{U}$ & $\mathcal{U}$ & 0 & $\{3\}$ & $\{3\}$ & $\emptyset$ & 1 \\
\hline$\{4\}$ & $\emptyset$ & $\mathcal{U}$ & $\mathcal{U}$ & 0 & $\{4\}$ & $\{4\}$ & $\emptyset$ & 1 \\
\hline$\{1,2\}$ & $\emptyset$ & $\mathcal{U}$ & $\mathcal{U}$ & 0 & $\{1,2\}$ & $\{1,2\}$ & $\emptyset$ & 1 \\
\hline$\{1,3\}$ & $\emptyset$ & $\mathcal{U}$ & $\mathcal{U}$ & 0 & $\{3\}$ & $\{1,2,3\}$ & $\{1,2\}$ & $\frac{1}{3}$ \\
\hline$\{1,4\}$ & $\emptyset$ & $\mathcal{U}$ & $\mathcal{U}$ & 0 & $\{4\}$ & $\{1,2,4\}$ & $\{1,2\}$ & $\frac{3}{3}$ \\
\hline$\{2,3\}$ & $\emptyset$ & $\mathcal{U}$ & $\mathcal{U}$ & 0 & $\{3\}$ & $\{1,2,3\}$ & $\{1,2\}$ & $\frac{1}{3}$ \\
\hline$\{2,4\}$ & $\emptyset$ & $\mathcal{U}$ & $\mathcal{U}$ & 0 & $\{4\}$ & $\{1,2,4\}$ & $\{1,2\}$ & $\frac{3}{3}$ \\
\hline$\{3,4\}$ & $\emptyset$ & $\mathcal{U}$ & $\mathcal{U}$ & 0 & $\{3,4\}$ & $\{3,4\}$ & $\emptyset$ & $\begin{array}{l}3 \\
1\end{array}$ \\
\hline$\{1,2,3\}$ & $\emptyset$ & $\mathcal{U}$ & $\mathcal{U}$ & 0 & $\{1,2,3\}$ & $\{1,2,3\}$ & $\emptyset$ & 1 \\
\hline$\{1,2,4\}$ & $\emptyset$ & $\mathcal{U}$ & $\mathcal{U}$ & 0 & $\{1,2,4\}$ & $\{1,2,4\}$ & $\emptyset$ & 1 \\
\hline$\{1,3,4\}$ & $\emptyset$ & $\mathcal{U}$ & $\mathcal{U}$ & 0 & $\{3,4\}$ & $\mathcal{U}$ & $\{1,2\}$ & $\frac{2}{4}$ \\
\hline$\{2,3,4\}$ & $\emptyset$ & $\mathcal{U}$ & $\mathcal{U}$ & 0 & $\{3,4\}$ & $\mathcal{U}$ & $\{1,2\}$ & $\frac{\frac{2}{4}}{4}$ \\
\hline $\mathcal{U}$ & $\mathcal{U}$ & $\mathcal{U}$ & $\emptyset$ & 1 & $\mathcal{U}$ & $\mathcal{U}$ & $\emptyset$ & 1 \\
\hline
\end{tabular}


From Table 8, for example, if we take $\{1,2,3\}$, then the similarity $10 j$-adhesion neighborhood boundary and the similarity $10 j$ adhesion neighborhood accuracy by Definition 4.17 are $\emptyset$ and 1 , respectively, whereas the similarity $4 j$-neighborhood boundary and the similarity $4 j$-neighborhood accuracy by using Dai et al.'s method [25] (as in Definition 2.8) (i.e., Definition 3.20 when $j=$ $j_{1}$ ) are $\{1,2,4\}$ and $\frac{1}{4}$, respectively. Also, from Table 9 , for example, if we take $\{3\}$, then the similarity $11 j$-adhesion neighborhood boundary and the similarity $11 j$-adhesion neighborhood accuracy by Definition 4.21 are $\emptyset$ and 1 , whereas the similarity $5 j$ neighborhood boundary and the similarity $5 j$-neighborhood accuracy by using Dai et al.'s method [25] (as in Definition 2.9) (i.e., Definition 3.25 when $j=j_{1}$ ) are $\{1,2,3\}$ and 0 , respectively. Additionally, from Table 10, for example, if we take $\{4\}$, then the similarity $12 j$-adhesion neighborhood boundary and the similarity $12 j$-adhesion neighborhood accuracy by Definition 4.25 are $\emptyset$ and 1 , whereas the similarity $6 j$-neighborhood boundary and the similarity $6 j$-neighborhood accuracy by using Dai et al.'s method [25] (as in Definition 2.10) (i.e., Definition 3.30 when $j=j_{1}$ ) are $\mathcal{U}$ and 0 , respectively.

From Tables 8-10, we can see the similarity 10-12 of lower and upper approximations based on $j$-adhesion neighborhood space are achieved to increase the accuracy measure and reduce the boundary region of set $\hat{X}$. Therefore, the results by Definitions 4.17, 4.21, and 4.25 when $j=j_{1}$ are better than the Dai et al.'s method [25] (as Definitions 2.8, 2.9, and 2.10) (i.e., Definitions $3.20,3.25$, and 3.30 when $j=j_{1}$ ), respectively.

\section{Conclusions}

In recent decades, many applications of the rough set theory have emerged in various fields. Therefore, in this paper, we aim to reduce the boundary region and increase the accuracy measure of decision-making. Besides, we introduce a generalization for six types of rough set models based on $j$-neighborhood space. A new six types of rough set models based on $j$-adhesion neighborhood space as, reflexive $7 j$-adhesion neighborhood rough set, reflexive $8 j$-adhesion neighborhood rough set, reflexive $9 j$ adhesion neighborhood rough set, similarity $10 j$-adhesion neighborhood rough set, similarity $11 j$-adhesion neighborhood rough set, and similarity $12 j$-adhesion neighborhood rough set are defined. Also, some of the fundamental properties of these types of rough $j$-adhesion neighborhood space are discussed. The relationship between the properties of different types is achieved. Finally, we made a comparison of previous methods (i.e., AboTabl's method and Dai et al.'s method) and our method of finding boundary region and accuracy. In the future, the present work can be extended to present some new rough set approximations under $j$-adhesion neighborhood space $[31,32]$.

\section{Acknowledgments}

The authors would like to thank editors and anonymous reviewers for their constructive comments, which help improve the paper significantly. This work was supported by the National Natural Science Foundations of China (11771263 and 61967001), the Fundamental Research Funds For the Central Universities (2018C BY001), the Key Research and Development Project of Shaanxi
Province of China (2018KW-050), and the Fundamental for Graduate students to participate in international academic conference (2018CBY001).

\section{Declarations}

\section{Conflict of interest}

All the authors certify that there is no conflict of interest with any individual or organization for the present work.

\section{Funding No funding.}

\section{Animal and human rights}

This article does not contain any studies with human participants or animals performed by any of the authors.

\section{References}

[1] Z. Pawlak, Rough sets, International Journal of Computer and Information Science, 11 (5) (1982), 341-356.

[2] Z. Pawlak, Rough concept analysis, Bulletin of the Polish Academy of Sciences Mathematics, 33 (1985), 9-10.

[3] M. Hosny and M. Raafat, On generalization of rough multiset via multiset ideals, Journal of Intelligent \& Fuzzy Systems 33 (2017), 1249-1261.

[4] S. Pal and P. Mitra, Case generation using rough sets with fuzzy representation, IEEE Transactions on Knowledge and Data Engineering, 16 (2004), 293-300.

[5] G. L. Liu and W. Zhu, The algebraic structures of generalized rough set theory, Information Sciences, 178 (2008), 4105-4113.

[6] K. Y. Huang, T.-H. Chang, T.-C. Chang, Determination of the threshold value $\beta$ of variable precision rough set by fuzzy algorithms, International Journal of Approximate Reasoning, 52 (7) (2011), 1056-1072.

[7] A. S. Salama, Topological solution of missing attribute values problem in incomplete information tables, Information Sciences, 180 (2010), 631639.

[8] Y. Y. Yao, Three-way decisions with probabilistic rough sets, Information Sciences, 180 (3) (2010), 341-353.

[9] S. Calegari, D. Ciucci, Granular computing applied to ontologies, International Journal of Approximate Reasoning, 51 (4) (2010), 391409.

[10] Q. Hu, L. Zhang, D. Chen, W. Pedrycz, D. Yu, Gaussian kernel based fuzzy rough sets: model, uncertainty measures and applications, International Journal of Approximate Reasoning, 51 (4) (2010), 453-471.

[11] Y. Qian, J. Liang, C. Dang, Knowledge structure, knowledge granulation and knowledge distance in a knowledge base, International Journal of Approximate Reasoning, 50 (1) (2009), 174-188.

[12] H. Zhang, H. Liang, D. Liu, Two new operators in rough set theory with applications to fuzzy sets, Information Sciences, 166 (1-4) (2004), 147-165.

[13] T. Herawan, M. Deris, J. Abawajy, Rough set approach for selecting clustering attribute, Knowledge-Based Systems, 23 (2010), 220-231.

[14] R. Jensen, Q. Shen, Semantics-preserving dimensionality reduction: rough and fuzzy-rough-based approaches, IEEE Transactions on Knowledge and Data Engineering, 16 (12) (2004), 1457-1471.

[15] X. P. Yang, T. J. Li, The minimization of axiom sets characterizing generalized approximation operators, Information Sciences, 176 (2006), 887 899.

[16] Y. Y. Yao Smarandache, Relational interpretations of neighbourhood operators and rough set approximation operators, Information Sciences, 111 (1998), 239-259.

[17] R. Slowinski, D. Vanderpooten, A generalized definition of rough approximations based on similarity, IEEE Transactions on Knowledge and Data Engineering, 12 (2) (2000), 331-336.

[18] A. Skowron, J. Stepaniuk, Tolerance approximation spaces, Fundamenta Informaticae, 27 (1996), 245-253.

[19] G. L. Liu, W. Zhu, The algebraic structures of generalized rough set theory, Information Sciences, 178 (21) (2008), 4105-4113.

[20] Y. Y. Yao, Constructive and algebraic methods of theory of rough sets, Information Sciences, 109 (1998), 21-47. 
[21] W. Zhu, Generalized rough sets based on relations, Information Sciences, 177 (22) (2007), 4997-5011.

[22] W. Zhu, Relationship between generalized rough sets based on binary relation and covering, Information Sciences, 179 (2009), 210-225.

[23] H. M. Abu-Donia, Comparison between different kinds of approximations by using a family of binary relations, Knowledge-Based Systems $\mathbf{2 1}$ (2008), 911-919.

[24] E. A. Abo-Tabl, A comparison of two kinds of definitions of rough approximations based on a similarity relation, Information Sciences, 181 (2011), 2587-2596.

[25] J. Dai, S. Gao, G. Zheng, Generalized rough set models determined by multiple neighbourhoods generated from a similarity relation, Soft Computing, 22 (2018), 2081-2094.

[26] M. E. Abd El-Monsef, O. A. Embaby, M. K. El-Bably, Comparison between rough set approximations based on different topologies, International Journal of Granular Computing, Rough Set and Intelligent Systems, 3 (2014), 292-305.

[27] M. I. Abbas, W. S. Amer, M. K. El-Bably, On j-near closure operators induced from relations and its applications, Cogent Mathematics, 3 (1247505) (2016), 1-17.

[28] M. Hosny, On generalization of rough sets by using two different methods, Journal of Intelligent \& Fuzzy Systems, 35 (1) (2018), 979-993.

[29] M. E. Abd El-Monsef, A. M. Kozae, M. K. El-Bably, On generalizing covering approximation space, Journal of the Egyptian Mathematical Society, 23 (2015), 535-545.

[30] W. S. Amer, M. I. Abbas, M. K. El-Bably, On j-near concepts in rough sets with some applications, Journal of Intelligent \& Fuzzy Systems, 32 (2017), 1089-1099.

[31] A. Kandil, S. A. El-Sheikh, M. Hosny, M. Raafat, Bi-ideal approximation spaces and their applications, Soft Computing, (2020). https://doi.org/10.1007/s00500-020-04720-2.

[32] H. M. Abu-Donia, Multi knowledge based rough approximations and applications, Knowledge-Based Systems, 26 (2012), 20-29. 